\title{
RADIAL VELOCITY DETECTION OF EARTH-MASS PLANETS IN THE PRESENCE OF ACTIVITY NOISE: THE CASE OF $\alpha$ CENTAURI Bb
}

\author{
Artie P. Hatzes \\ artie@tls-tautenburg.de \\ Thüringer Landessternwarte, D - 07778 Tautenburg, Germany
}

Received — ; accepted 


\begin{abstract}
We present an analysis of the publicly available HARPS radial velocity (RV) measurements for $\alpha$ Cen B, a star hosting an Earth-mass planet candidate in a 3.24 day orbit. The goal is to devise robust ways of extracting low-amplitude RV signals of low mass planets in the presence of activity noise. Two approaches were used to remove the stellar activity signal which dominates the RV variations: 1) Fourier component analysis (pre-whitening), and 2) local trend filtering (LTF) of the activity using short time windows of the data. The Fourier procedure results in a signal at $P=3.236 \mathrm{~d}$ and $K=0.42 \mathrm{~m} \mathrm{~s}^{-1}$ which is consistent with the presence of an Earth-mass planet, but the false alarm probability for this signal is rather high at a few percent. The LTF results in no significant detection of the planet signal, although it is possible to detect a marginal planet signal with this method using a different choice of time windows and fitting functions. However, even in this case the significance of the 3.24-d signal depends on the details of how a time window containing only $10 \%$ of the data is filtered. Both methods should have detected the presence of $\alpha \mathrm{Cen} \mathrm{Bb}$ at a higher significance than is actually seen. We also investigated the influence of random noise with a standard deviation comparable to the HARPS data and sampled in the same way. The distribution of the noise peaks in the period range $2.8-3.3 \mathrm{~d}$ have a maximum of $\approx 3.2 \mathrm{~d}$ and amplitudes approximately one-half of the $K$-amplitude for the planet. The presence of the activity signal may boost the velocity amplitude of these signals to values comparable to the planet. It may be premature to attribute the 3.24 day RV variations to an Earth-mass planet. A better understanding of the noise characteristics in the RV data as well as more measurements with better sampling will be needed to confirm this exoplanet.
\end{abstract}


Subject headings: planetary systems — techniques: radial velocities 


\section{Introduction}

Precise stellar radial velocity (RV) measurements can currently achieve a precision better than a few $\mathrm{ms}^{-1}$ and this has enabled astronomers to detect planets with masses of just a few Earth masses (e.g. Tuomi et al. 2013; Mayor et al. 2009). The ability of the RV method to detect the lower mass planets $\left(\approx 1 M_{\oplus}\right.$ or smaller) hinges not on the measurement error provided by instruments, but rather on the 'error' of the intrinsic stellar variability. With a precision of below $1 \mathrm{~ms}^{-1}$ (Pepe et al. 2011) RV measurements are approaching the stellar noise floor of many solar-type stars.

The RV 'jitter' caused by magnetic activity (spots, plage, changes in the convection pattern, etc.) is a major source of stellar noise that can hinder the detection of Earth-mass planets. For example, a spot coverage of only $0.5 \%$, typical for the sun at solar maximum, can induce an $R V$ variation of $\approx 0.5 \mathrm{~m} \mathrm{~s}^{-1}$ (Saar \& Donahue 1997; Hatzes 2002). This is the velocity amplitude of the stellar reflex motion caused by an Earth-mass planet around Sun-like star with an orbital period of a few days. An Earth-mass planet 1 AU from the star will cause an RV motion of a mere $0.09 \mathrm{~m} \mathrm{~s}^{-1}$.

In order to detect Earth-mass planets with RV measurements around solar-type stars we must devise ways of overcoming the activity noise. This will be challenging as these activity RV variations, depending on the spot distribution on the stellar surface, will be modulated by the rotation period, $P_{\text {rot }}$, of the star as well as its higher harmonics $\left(P_{\text {rot }} / 2\right.$, $P_{\text {rot }} / 3, P_{\text {rot }} / 4$, etc.). Spot evolution and activity cycles coupled with the sampling window will add other frequencies to the power spectrum of the RV variations. These intrinsic variations must be filtered out in a robust way.

Dumusque et al (2012; hereafter D2012) demonstrated that it may be possible to 'break the activity barrier' and detect an Earth-mass planet in the presence of stellar activity. The authors used RV measurements to find a $1.13 \pm 0.09 \mathrm{M}_{\oplus}$ planet with a 3.236 day orbital 
period around $\alpha$ Cen B. The discovery of this low mass planet was challenging. Alpha Centauri B has a modest level of activity that creates substantial RV 'jitter' compared to the planet signal. The RV measurements showed a dominant periodic signal at $\approx$ 38 days with an amplitude of $\approx 1.5 \mathrm{~ms}^{-1}$ or at least three times the so-called velocity $K$-amplitude caused by the planet. To remove this activity signal D2012 used a harmonic filtering approach. In this method RV measurements are selected using a time interval of a few rotational periods and fitting the RV activity variations with sine functions using the rotational frequency, $\nu_{\text {rot }}$, and its harmonics $\left(2 \nu_{\text {rot }}, 3 \nu_{\text {rot }}\right.$, etc.). If the peak in the periodogram had a false alarm probability less than $10 \%$ and its period was equal to the rotational period or one of its harmonics it was removed. The harmonic method has been used to detect the RV variations of the transiting rocky planet CoRoT-7b (Queloz et al. 2009; Ferraz-Mello et al. 2011) as well as to reduce the activity noise of other planet hosting stars (Boisse et al. 2011).

The RV detection of the rocky planet CoRoT-7b also demonstrated that a planetary signal can be extracted from RV measurements dominated by variations due to activity. The detection of CoRoT-7b was easier than $\alpha$ Cen Bb for a number of reasons: 1) although the $K$-amplitude of the star due to the companion $\left(\approx 5 \mathrm{~m} \mathrm{~s}^{-1}\right)$ was a factor of two smaller than the activity variations, it was still larger then the measurement error of $\approx 2 \mathrm{~m} \mathrm{~s}^{-1}$. 2) The RV data were taken over a relatively short time span of a few months. 3) The orbital period of the planet was already known from transit light curves (Léger et al. 2009). 4) Finally, the orbital period was much smaller than the rotational period of the star by a factor of almost 30 .

The detection of $\alpha$ Cen $\mathrm{Bb}$ posed more challenges compared to CoRoT-7b: 1) the stellar $K$-amplitude $\left(\approx 0.5 \mathrm{~m} \mathrm{~s}^{-1}\right)$ is comparable, if not smaller than the measurement error and smaller than the intrinsic stellar variations. 2) To detect the planet signal D2012 had 
to combine data taken over three years, thus spot evolution and activity cycles may be more of a problem than it was for CoRoT-7b. 3) The period of the planetary companion was not known a priori and it requires significantly more data to detect an unknown period in a time series, particularly when the amplitude is comparable to the measurement error. 4) The planet period was a relatively long 3.236 days which is a factor of 10 shorter than the rotational period of the star. Alpha Cen B thus represents a different and more difficult case than CoRoT-7b for the detection of planetary signal in the presence of stellar noise. As noted by Hatzes (2012), the large number of the RV measurements for $\alpha$ Cen B as well as their high quality provides an excellent data set for astronomers to test their analysis tools for extracting planetary signals in the presence of stellar activity.

In this paper we analyze the HARPS RV data for $\alpha$ Cen B using several approaches to filter out the activity noise. There are several goals to this investigation: 1) to test the effectiveness of various approaches to filtering out the activity. 2) To check the robustness of the planet signal. If it can be detected with different methods then we can be more confident of its presence. 3) To obtain a better determination of the planet mass (i.e. $K$-amplitude). CoRoT-7b demonstrated that the various ways of filtering the activity resulted in masses for the transiting rocky planet that initially differed by factors of four (see Hatzes et al. 2011). A different analysis may produce a revised mass for $\alpha$ Cen Bb.

In most planet detections using RV data researchers rely primarily on the Lomb-Scargle periodogram and few use the discrete Fourier transform (DFT) which is primarily employed by the stellar oscillation community. In this work both tools will be employed as each has its purpose. We use the DFT to understand the velocity amplitude of various signals that are present in the data and to detect and subsequently remove the dominant Fourier components (sine functions). The Scargle (1982) periodogram is used primarily because it gives a measure of the statistical significance of a periodic signal. 
Since both the DFT and the Scargle periodogram will be employed in this paper it is worthwhile commenting about the differences between the two. In DFT analysis the power is in units of $\left(\mathrm{m} \mathrm{s}^{-1}\right)^{2}$. In this paper the amplitude is shown so that the reader can readily assess the velocity amplitude of a signal as well as the true velocity noise floor surrounding a peak. For a DFT the amplitude of a signal remains constant regardless of the number of data points or significance of a signal. For a real signal, the acquisition of additional data does not change the amplitude significantly, it merely reduces the overall noise floor in Fourier space. On the other hand, the power in a Scargle periodogram is a measure of the statistical significance of a signal. For a real signal as you acquire more data the significance of the detection increases and so does the Scargle power, but in a non-linear way. As a rule-of-thumb for the periodograms shown here, Scargle power, $z$, less than 10 is most likely not a significant signal, $10<z<14$ is a modestly significant signal that is interesting and merits more investigation (i.e. an improved analysis or more data), whereas $z>20$ is most likely a true signal.

In the exoplanet community it has become a common practice to show periodograms using period for the abscissa. In this work a frequency scale will be used for two reasons. First, frequency is the natural units when using a DFT or periodogram (in spite of the name). Second, the use of period distorts the periodogram making it difficult to judge the comparative width of features as well as the noise floor surrounding a peak. When

appropriate both period and frequency will be given. Frequency will be given in units of $\mathrm{d}^{-1}$ rather than $\mathrm{Hz}$ since day is the unit often used to to express orbital periods for exoplanets.

\section{The Radial Velocity Data}

The high precision RV measurements used for this analysis are the ones presented by D2012. These were taken with the HARPS spectrograph at the 3.6m telescope at La Silla 
Observatory of the European Southern Observatory. A total of 459 RV measurements were made between 2008 February and 2011 July. The RV measurements had a median photon error of $0.4 \mathrm{~m} \mathrm{~s}^{-1}$ and D2012 estimated a systematic error of $0.7 \mathrm{~m} \mathrm{~s}^{-1}$. Adding these in quadrature results in a total error of about $0.8 \mathrm{~m} \mathrm{~s}^{-1}$. We take this as the "best case" estimate of the RV error. More details of the data analysis and reduction can be found in D2012.

The RV measurements for $\alpha$ Centauri B showed a long term trend that is part of the binary orbital motion with component A. This orbital motion was removed by fitting it with a second order polynomial as was done in D2012. This should be adequate since the rotational period of the star and orbital period of the planet are both considerably less than the binary orbital period of several decades. Throughout the paper the term "RV data" will refer to the RV measurements after removal of only the binary orbital motion. The term 'RV residuals' will refer to RV data that has all variations presumably due to activity removed, but with any 'planetary' signal still in the data.

The HARPS data were taken over four epochs spanning more than three years. Table 1 lists the Julian day (JD) span of the epoch, the time span in days, the number of measurements, $N_{\text {obs }}$, made in that epoch, and the standard deviation, $\sigma$, of the RVs after removing the orbital motion. Throughout this paper JD values will be given as reduced Julian day $(\mathrm{RJD}=\mathrm{JD}-2400000)$. Epoch 1 has a standard deviation only slightly more than the estimated error indicating a low level of activity for the star, as noted by D2012. Epoch 3 has the largest standard deviation which implies a more active phase of the star. 


\section{Results}

\subsection{Fourier Component Analysis Via Pre-whitening}

Pre-whitening is a commonly used tool for finding multi-periodic signals in time series data. This method sequentially finds the dominant Fourier components in a time series and removes them. Traditionally it is employed to derive the frequency spectrum of oscillating stars (e.g. García Hernández et al. 2009), but it also has applications as a means of removing the intrinsic stellar variations due to activity. The mathematical foundation for this is that sines and cosines form a set of basis functions. You can represent most functions as a sum of sine waves with different periods and amplitudes. In fact, the DFT of a time series merely gives you the amplitude as a function of frequency of all the sine functions that are present, including those due to noise. The trick is to use enough Fourier components to represent adequately the function of interest (activity in this case) without introducing spurious periods, or altering the amplitude and frequency of a signal you are trying to detect. So long as artifacts introduced by the multi-component sine fit have frequencies and amplitudes different from our signal of interest, pre-whitening can be a useful tool for detecting weak periodic signals. In this case we have the advantage in that we have a priori information about the signal we are trying to detect.

The pre-whitening process is similar to the harmonic analysis used by of D2012 in that both fit the activity signal with multi-sine components. There are, however, two major differences. First, in pre-whitening the time span is not restricted to a few rotational periods, but in our case we use a much longer time span. Second, the frequencies that are removed by pre-whitening are not restricted to just the rotational frequency and its harmonics. The strongest peak in the DFT is removed regardless of its frequency. One can consider harmonic analysis as a more restricted version of pre-whitening. 
The case of CoRoT-7b demonstrated the effectiveness of Fourier component analysis in fitting an activity signal. The RV activity jitter for CoRoT-7 was about a factor of 10 higher than for $\alpha$ Cen B. The pre-whitening process resulted in a $K$-amplitude of $5.5 \pm$ $0.3 \mathrm{~m} \mathrm{~s}^{-1}$ (Hatzes et al. 2010). This was consistent with the value of $K=5.27 \pm 0.81$ $\mathrm{m} \mathrm{s}^{-1}$ determined using an entirely different filtering approach that did not rely on periodic functions (Hatzes et al. 2011). On the other hand, the initial $K$ amplitude derived from harmonic analysis was $1.9 \mathrm{~m} \mathrm{~s}^{-1}$, or almost a factor of three lower than the final value (Queloz et al. 2009). Furthermore, the harmonic analysis failed to detect the presence of a third planetary companion, CoRoT-7d (Hatzes et al. 2010).

In pre-whitening process one usually picks the highest peak in the Fourier amplitude spectrum of the time series. A least squares sine fit is made to the data to determine the optimal frequency, amplitude, and phase. This fit is then subtracted from the data which also removes (or at least minimizes) the effects of alias peaks caused by this signal. A Fourier analysis on the residuals then finds the next dominant peak and the process is continued until one reaches the noise level of the amplitude spectrum. Each process thus "whitens" the data in frequency space for the next step. The resulting frequencies that are found should represent the dominant frequency components of the time series. In our case we use the sum of the pre-whitened components we have found to provide us with a fit to the activity variations.

The Fourier components one derives (amplitude, frequency, and phase) depend on the length of the time series and the presence of time gaps (sampling window). Some of the effects of these can be explored by analyzing first the complete data set, and then subsets divided into epochs of the measurements. In doing so one may derive slightly different Fourier components in fitting the underlying activity variations. A robust signal should be relatively insensitive as to how we pre-whiten the data. 


\subsubsection{Fourier Component Analysis of the Full Data Set}

The pre-whitening procedure was applied to the full RV data using the program Period04 (Lenz \& Breger 2004). For clarity we only show the DFT of the un-whitened data (top panel Figure 1) rather than each step of the process. In the figure we have marked the frequencies found by the pre-whitening process. Note that removing a peak also removes its alias in Fourier spectrum whose amplitude may be higher than a real peak. The amplitude spectrum is dominated by a forest of peaks in the frequency range $0<\nu<0.1 \mathrm{~d}^{-1}$. These may be due to the rotational frequency of the star, its harmonics, as well as the spectral window (sampling). The highest peaks corresponds to a frequency $\nu=0.025 \mathrm{~d}^{-1}$ (period, $P=38 \mathrm{~d}$ ) which is interpreted as the rotational frequency of the star. This frequency has

an amplitude of $\approx 1.5 \mathrm{~m} \mathrm{~s}^{-1}$ which is about three times larger than the $K$-amplitude of the purported planet. Note that the orbital frequency at $\nu=0.309 \mathrm{~d}^{-1}$ is hardly visible in the original un-whitened data.

Table 2 lists the frequencies, corresponding periods, amplitudes, and phases for all signals found in the RV time series. Note that amplitudes found in Table 2 may differ slightly to those seen in Figure 1 due to removal of peaks and their aliases. The lower panel of Figure 1 shows the final pre-whitened amplitude spectrum with only the peak near the orbital frequency of $\alpha \mathrm{Cen} \mathrm{Bb}\left(f_{9}\right)$ remaining. Note that in fitting the data simultaneously with all frequencies a slightly higher amplitude $\left(\approx 1.9 \mathrm{~m} \mathrm{~s}^{-1}\right)$ for the rotational frequency results when compared to the initial DFT. Removing all frequencies in Table 2 from the RV data results in a standard deviation of $1.17 \mathrm{~m} \mathrm{~s}^{-1}$. Interestingly, this value of $\sigma$ is consistent with the rms scatter of Epoch 1 for which D2012 noted was a time when the star was relatively inactive and thus should have a lower RV jitter. We thus use $\sigma=1.2 \mathrm{~m} \mathrm{~s}^{-1}$ as our "worse case" estimate of the RV error.

The low frequency component $\left(f_{2}\right)$ has a period, $763 \mathrm{~d}$, that is shorter than the time 
span of the observations of $1230 \mathrm{~d}$. This is most likely due to some variation of the activity, but we cannot exclude with certainty that it may be caused by slight differences between the parabolic fit and the true Keplerian orbit. Unfortunately due to the long period this orbit is poorly known. Because the period is over a factor of 200 greater than the planet orbital period this should not effect the subsequent analysis, particularly for the local trend fitting analysis (see below). All errors are uncorrelated errors from Period04; correlated errors are most certainly larger. Many frequencies can be identified with the rotational frequency, $\nu_{\text {rot }}$, or its harmonics: $f_{1}=\nu_{\text {rot }}, f_{3}, f_{8} \approx 3 \nu_{\text {rot }}, f_{4}=4 \nu_{\text {rot }}$, and $f_{6} \approx 2 \nu_{\text {rot }}$. This gives some justification to the harmonic analysis used by D2012. Note that the last entry $\left(f_{9}\right)$ in the table corresponds to the orbital frequency of $\alpha$ Cen Bb. The pre-whitened period $(P=3.2356 \pm 0.0001 \mathrm{~d})$ is identical to the value $P=3.2357 \pm 0.0008 \mathrm{~d}$ found by D2012. The amplitude, $K=0.40 \pm 0.08 \mathrm{~m} \mathrm{~s}^{-1}$, is bit lower, but still consistent within the errors to the value of $K=0.51 \pm 0.04 \mathrm{~m} \mathrm{~s}^{-1}$ from D2012. The implied companion mass from the pre-whitened amplitude is $m=0.89 \pm 0.18 \mathrm{M}_{\oplus}$.

The statistical significance of the $3.24 \mathrm{~d}$ signal was assessed with a Scargle periodogram analysis (top panel of Fig. 2) of the residual RV data produced after removing the first eight frequencies listed in Table 1 . The peak at the planet orbital frequency of $0.309 \mathrm{~d}^{-1}(P$ $=3.24 \mathrm{~d})$ appears to be significant due to its high Scargle power $(z \approx 12)$. The false alarm probability (FAP) of this peak was assessed using the bootstrap randomization process. The residual RV values were randomly shuffled while keeping the time values fixed. The highest peak in the Scargle periodogram in the frequency range $0.0001<\nu<0.5 \mathrm{~d}^{-1}$ was found for each random data set. The number of instances where the shuffled data produced power higher than the observed power provided a measure of the FAP. The resulting FAP was $\approx 0.004$. (Note that all FAP values given below are the result of bootstrap analyses performed with 200000 shuffles.) 
It is difficult to assess the significance of a periodic signal in the presence of other signals (in this case at least eight) that have been filtered from the data. The FAP calculated with a bootstrap depends on the scatter in the data. After removing a periodic signal the amount of scatter in the data is reduced and a much lower FAP may thus result. However, one cannot be sure that a peak in the amplitude spectrum is a true signal and should be removed (e.g., rotation), or a noise peak that should remain in the data when performing a FAP analysis. The bootstrap analysis may produce an unrealistically low FAP simply because you have "cleaned" the data by lowering the noise floor in Fourier space. An alternative approach is to use the unfiltered amplitude spectrum itself. Kuschnig et al. (1997) established that peaks in the amplitude spectrum that have a height 3.6 times the surrounding noise level corresponds to a $\mathrm{FAP} \approx 1 \%$.

Period04 calculates a noise level at the orbital frequency of the planet of $0.23 \mathrm{~m} \mathrm{~s}^{-1}$. The amplitude of the planet signal is $\approx 0.4 \mathrm{~m} \mathrm{~s}^{-1}$ which is only a factor of 1.7 above the noise level. This corresponds to a FAP of $\approx 100 \%$. If one uses the pre-whitened amplitude spectrum with all frequencies in Table 2 removed except for the planet orbital frequency the noise level is $0.12 \mathrm{~m} \mathrm{~s}^{-1}$. This is the same value as simply taking the mean amplitude of peaks over the interval $0.25 \mathrm{~d}^{-1}<\nu<0.35 \mathrm{~d}^{-1}$. This amplitude is 3.3 times the noise level which corresponds to a FAP $\approx 5 \%$. The noise level of the DFT amplitude spectrum thus indicates a FAP of approximately a few percent, but with a large uncertainty. Given the complexity of the RV variations it is difficult to assess an accurate false alarm probability.

The efficacy of the Fourier procedure was tested on simulated data. First, the 3.236- day period Fourier component (last entry in Table 2) was removed from the HARPS RV data on the assumption that this signal is real. The orbital solution of D2012 was then added back into the data. We also added synthetic planet signals at slightly different orbital periods. For these other simulations we used the RV data without removing the $3.236 \mathrm{~d}$ 
component on the assumption that this is due purely to noise in the data. In this way all simulations preserved the noise characteristics of the real data. As an example, the lower panel in Figure 2 shows a Scargle periodogram of the residual RV data with an artificial planet inserted prior to filtering the data. The planet signal was taken as a simple sine wave with a period of $3.29 \mathrm{~d}$ (slightly different from the period of $\alpha$ Cen $\mathrm{Bb}$ ) and an amplitude of $0.5 \mathrm{~m} \mathrm{~s}^{-1}$.

Table 3 summarizes the results of the Fourier filtering of the simulated data. The third entry is the simulation using the orbital parameters from D2012. The table lists the input period of the sine function, $P_{i n}$, the input amplitude, $K_{i n}$, the period recovered by the pre-whitening procedure, $P_{\text {out }}$, the output amplitude $K_{\text {out }}$, and the FAP of the detected signal computed using a bootstrap. In all cases the input period and amplitude are recovered well and at high level of significance.

\subsubsection{Fourier Component Analysis of the Individual Epochs}

The pre-whitening procedure was then applied to the individual epochs in Table 1 . In cases where you have periodic signals that are evolving with time (e.g. the birth, decay, evolution, and migration of surface spots) pre-whitening of a long time series with large gaps may produce poorer results. A much better fit to the activity could be obtained by using data covering a shorter time interval. Furthermore, since one is deriving a different set of sine functions for filtering the data, this is an independent check on how robust the signal that was found when analyzing the full data set is.

Figure 3 shows the fit to the activity variations to each of the epochs and Table 4 lists the sine parameters. The first subscript in the frequency identification (ID) refers to the epoch number from Table 2. The DFTs of the RVs from the individual epochs are shown 
in Figure 4. In the figure we have marked the frequencies found by pre-whitening the data. In Epoch 2 the second dominant peak was found at $0.356 \mathrm{~d}^{-1}(P=2.8 \mathrm{~d})$. This was not removed since it has a frequency near that of the planet orbital frequency.

The upper panel of Figure 5 shows the periodogram of the total RV residuals from all epochs. The epoch pre-whitened RV residuals have a standard deviation of $\sigma=1.16$ $\mathrm{ms}^{-1}$ with the planet signal present. One can still see a peak at the orbital frequency of the planet, but the power is reduced from that found by the analysis on the full data set. A bootstrap analysis yields a FAP of only 0.07 for this peak. Removing the planet signal reduces the standard deviation slightly to $\sigma=1.13 \mathrm{~m} \mathrm{~s}^{-1}$.

The epoch pre-whitening was also tested on simulated data. A sine wave $(P=3.29 \mathrm{~d}$, $\nu=0.304 \mathrm{~d}^{-1}, K=0.5 \mathrm{~m} \mathrm{~s}^{-1}$ ) was inserted in the data prior to pre-whitening. The lower panel of Fig. 5 shows the Scargle periodogram of the residual RV data. The FAP of this signal is $<5 \times 10^{-6}$ based on a bootstrap analysis. As another test a sine fit to the 3.24 day period was made to the full data set and removed (i.e. removal of $f_{9}$ from Table 2 ) and the orbital solution from D2012 inserted back into the data. The pre-whitening procedure was then applied to the epoch data. The planet signal was detected with a FAP $=0.01 \%$.

The pre-whitening process on the epoch data was able to detect the planet, but with much reduced significance. One would naively think that since essentially the same technique is applied to both the full and epoch data that we should arrive at the same answer. Indeed, we removed from the data the 3.24-d period found in pre-whitening the full data set and re-inserted the orbital solution for $\alpha$ Cen Bb from D2012. Pre-whitening of the epoch data showed significant Scargle power of $z \approx 15$ which corresponds to FAP $\approx 0.03$ $\%$. This is consistent with the full data set pre-whitening: $z \approx 18, \mathrm{FAP} \approx 0.002 \%$. Both approaches to filtering the data detects the planet with a much higher significance than was found. The discrepancy is the first hint that the planet signal may depend sensitively on 
how the data is filtered. Clearly, an independent, non-Fourier based technique is needed to help resolve this discrepancy.

\subsection{Local Trend fitting}

Although Fourier pre-whitening is a useful tool for getting a quick result, it has its drawbacks. Some functions, like a linear trend, may require a large number of Fourier components (i.e. free parameters) to fit them when simpler functions with fewer free parameters such as low order polynomials could provide a better fit. Furthermore, with multi-sine components, if one uses insufficient Fourier components there can be mis-match between the true activity variations and the fit. The sampling window complicates matters further. All of these may introduce false peaks in the filtered data, or increase the apparent significance of noise peaks. It is therefore important to check the results of pre-whitening and harmonic analysis by using alternative filtering techniques that rely less on periodic functions and that have fewer free parameters. For a robust signal, different filtering approaches should produce comparable results as was the case for CoRoT-7b.

A better filter should exploit the fact that we know the periods of interest, namely the orbital period of the planet as well as the rotation period of the star. If we can fit the activity variations over a much shorter time span the signal should be more coherent and stable. We thus should be able to use functions with fewer parameters that fit better the activity variations at that particular time as opposed to using a global fit that requires more free parameters (sine functions). Trend filtering is often employed to remove the stellar variations when searching for transit signals in light curves (Kovács et al. 2005; Grziwa et al. 2012; Bakos et al. 2013).

The time window for fitting the activity variations is bounded by two limits. The lower 
limit is the orbital period of the planet. Filtering out activity variations over a time span less than this runs the risk of suppressing any real RV variations due to the planet. The upper limit is defined by the rotation period of the star, a time span over which we consider the activity variations likely to be stable and coherent.

The RV data were visually inspected and divided into time chunks for the local trend fitting. The following criteria were used to decide which data went into a specific chunk: 1) the time interval of a chunk, $\Delta T$ should cover as many cycles of the planet orbit as possible but at least a full orbital period, but less than a stellar rotation period: $P_{\text {orbit }}$ $<\Delta T<P_{\text {rot }}$. 2) The time series should have good sampling (preferably nightly) in the interval and with no gaps longer than a day or two. 3) In fitting trends the data should be grouped to avoid large gags in temporal coverage or abrupt changes in the long term variations in the chunk. 4) If successive measurements were separated by several days, but seemed to follow the overall trend they were kept in the analysis. If they showed significant departures from the trend that required more complicated fitting functions (i.e. a high order polynomial with more inflections), they were removed. In total 41 data points were removed from the original HARPS data. In short, the data were grouped in subsets that showed smooth variations of the underlying trend. The fit to these should have different Fourier components that are far removed in frequency from those of the planetary signal.

The choice of fitting function for the trend in each chunk was determined visually so that the fit to the underlying activity variations would have a minimal influence on the shorter periodic variations of the planet. If the data in a chunk showed no long term variations, the average value was subtracted. If it showed a linear trend a least squares linear fit was made. In cases where the chunk trend showed curvature a second or third order polynomial were used. Although we tried to avoid using periodic functions, in one chunk the underlying activity variations could best be fit with a multi-sine component (see 
below).

Two time intervals RJD $=54935-54955$ and RJD $=55672-55692$ showed significant periodic variations. In the first of these intervals the RV data centered on RJD $=54939.68$ - 54941.85 showed an additional linear trend on top of the sinusoidal variations (look ahead to the lower left panel of Figure 12). Since there was a large gap of five days with the first group of points in this interval these 12 points were removed (in the lower left panel of Figure 12 these are marked by the bracket). This ensured a simpler fitting function with fewer inflections. (In $\S 3.4$ we will see that this one time chunk can have a large influence on the amplitude spectrum.) The time interval RJD $=54935-54955$ was thus divided into two chunks. In the first (chunk \#8) a second order polynomial was used to fit the trend, and a third order polynomial for the next chunk (chunk \#9).

The interval RJD $=55672-55692$ had good sampling and the data looked periodic. Two sine functions with $\nu=0.043 \mathrm{~d}^{-1}, K=1.2 \mathrm{~m} \mathrm{~s}^{-1}$, and $\nu=0.08 \mathrm{~d}^{-1}, K=2.3 \mathrm{~m} \mathrm{~s}^{-1}$, values found by pre-whitening the data were used to fit the activity variations. These data comprised chunk \#20.

Table 5 lists the Julian day of the time chunks, the time span, $\Delta T$, the number of planet orbits during this span, $N_{\text {orb }}$, the number of data points used in the fit, $N_{\text {data }}$, the fitting function employed (constant $=$ average value subtracted, linear, second or third order polynomial), and the rms scatter in the chunk, $\sigma$, after removing the trend but with the planet signal present. Figures 68 show the individual time chunks used in the analysis of the HARPS data. The error bars represent the best case error of $0.8 \mathrm{~m} \mathrm{~s}^{-1}$. The solid lines represent the fit to the underlying trend in the chunk. As comparison, the fit to the activity using the sine parameters found by pre-whitening the full data set (Table 2, but without the planet contribution) is also shown. Note that there are many instances where the LTF provides a much better fit to the underlying activity variations. 
The residual RVs after removing the underlying trend were then combined. These had a standard deviation of $\sigma=0.94 \mathrm{~m} \mathrm{~s}^{-1}$. The Scargle periodogram (top panel Figure 9) shows no significant peak anywhere in the frequency range $\nu=0-0.5 \mathrm{~d}^{-1}$. The peak at the planet orbital frequency is weak and has a FAP of $\approx 0.4$ as determined by a bootstrap. As a quick test the Fourier component at 3.24-d $\left(\nu=0.309 \mathrm{~d}^{-1}, f_{9}\right.$ in Table 2$)$ was removed from the full data set and the orbit of $\alpha$ Cen Bb of D2012 added back to the data. The data with the simulated planet were divided into chunks and trend filtered as the previous data, and calculating new trends for the data. The Scargle periodogram of the residuals (lower panel Figure 9) shows that the planet signal should have been easily detected and with a FAP $\approx 0.01 \%$. The local trend filtering method does not confirm the presence of a planetary signal at $3.235 d$ around $\alpha$ Cen $B$.

\subsection{Tests of the Local Trend Filtering Procedure}

The local trend filtering (LTF) procedure was tested further to see how well it could recover known signals in simulated data generated in a different ways. This was done on three different simulated data sets:

1. The 3.24 day planet period was removed from the RV data using the sine function parameters found by the pre-whitening process. A sine function with the same amplitude as the planet $\left(0.5 \mathrm{~m} \mathrm{~s}^{-1}\right)$ but with a slightly different period, $P=3.27 \mathrm{~d}$, was inserted back into the data. A different period was employed simply to avoid the frequency in the amplitude spectrum where signal was removed. This simulation keps the original noise characteristics of the data.

2. A signal with a period of 3.37 days and a $K$-amplitude of $0.5 \mathrm{~m} \mathrm{~s}^{-1}$ was inserted into the RV data. A different period to that of the planet was used because this possible 
signal is still in the data and we want to avoid interference between the two. (As far as LTF is concerned a 3.37 day period is no different from a 3.23 day period.) In this simulation all the noise characteristics of the data are kept, and with the assumption that the signal at 3.24 days is also due to noise.

3. A simulated activity signal was generated using the first eight frequencies, amplitudes, and phases listed in Table 2. (Hereafter we will refer to this simulated activity signal as "the activity function".) This was then sampled in the same way as the data and random noise with standard deviation, $\sigma=0.8 \mathrm{~m} \mathrm{~s}^{-1}$ was added. The orbit of D2012 was then inserted into the data. The advantage of this simulation is that the underlying trend for each chunk may be slightly different to the cases above. The disadvantage is that the noise characteristics, which are now Gaussian, may be different than for the real data.

The LTF technique was applied to each of these three artificial data sets. Although the time span for each chunk was kept fixed, the fit to RV data was repeated in each case. Scargle periodograms of the trend-removed residuals (Figure 10) show that the local filtering process was able to recover the input signal and at a high significance in all cases. A bootstrap analysis with 200000 shuffles showed no instance where the periodogram of random data exceeded the real periodogram $\left(\mathrm{FAP}<5 \times 10^{-6}\right)$. Note that these signals were detected at a much higher level of significance than the original data. Tests using planet orbital variations with a different phase produced similar results. It appears that the filtering process is not suppressing a possible real signal from a planet.

The last simulation assumed our best case estimate of the noise. It is of interest to explore the detection limits of the LTF procedure as a function of different noise levels. Again synthetic data consisting of the activity function plus the planet orbit parameters of D2012 were used, but this time with different levels of random noise added. Figure 11 
shows the Scargle power of the RV residuals as a function of the standard deviation, $\sigma$, of the random noise. Shown are simulations for three values of the $K$-amplitude $(0.3,0.4$, and $\left.0.5 \mathrm{~m} \mathrm{~s}^{-1}\right)$. If the real measurement error is close the best case value a $K$-amplitude of $\approx$ $0.35 \mathrm{~m} \mathrm{~s}^{-1}$ could be detected with a FAP $=1 \%$. For a $K$-amplitude of $0.5 \mathrm{~m} \mathrm{~s}^{-1}$ the planet could be detected at the $1 \%$ level even for $\sigma$ as high as $\approx 1.4 \mathrm{~m} \mathrm{~s}^{-1}$ For planets with certain orbital periods it should be possible to overcome the activity noise.

The LTF method was also used with different time windows and fitting functions to explore as in the case of pre-whitening how different filters could influence the results. A second trend filtered version of the data (hereafter LTF2) was made with the following minor differences compared to previous version of the trend filtering (hereafter LTF1):

1. In LTF1 the data taken during RJD $=54549$ - 54572 were divided into two chunks because of a 5-day gap. A linear fit to each was made separately (chunks \#2 and \#3). In LTF2 a parabola was fit to all the data (upper right panel of Figure 12) across the gap.

2. In chunk \#6 of LTF1 a parabola was fit to the data, but after removing the last data points that came after a 2-day gap. In LTF2 these points were included, but it forced one to use a higher order polynomial (top right panel of Figure 12).

3. For measurements made during RJD $=54933-54956$ the data were divided into two chunks (chunks \#8 and \#9) in LFT1 because of a five day gap in the time sampling. In LTF2 data in chunks \#8 and \#9 were combined and a multi-component sine function was fit to the underlying trend throughout the time interval (lower left panel of Figure 12.

4. For chunk \#20 an additional sine component was used as determined from pre-whitening the data (lower right panel Figure 12) 
Figure 12 shows the new trend fits to these time chunks. The total rms scatter of the data (including the other chunks) after removing the trends is about $1.00 \mathrm{~m} \mathrm{~s}^{-1}$, or slightly worse than for LTF1. For all the other chunks the same time windows and fitting functions were employed as per LTF1. The periodogram of the combined trend-removed residuals shows modest power power $(\mathrm{FAP} \approx 0.006)$ at the frequency near with the orbital frequency of the planet (top panel of Figure 13). (Also, the highest peak occurs at a slightly different frequency, $\nu=0.30615 \mathrm{~d}^{-1}$, or $P=3.26$ days). However, it is highly suspicious that a markedly different periodogram is obtained when altering slightly the fit to a small subset of the data. This simulation only reinforces what was found in performing the pre-whitening on the full and epoch data sets - slight differences in the way the RV data is filtered can produce dramatically different results.

\subsection{The Significance of the LTF2 Detection}

In the course of filtering the activity signal from the individual chunks we discovered that data in the time window JD-2400000 $=54933-54956$ (hereafter referred to as "Chunk8-9" since it is a combination of chunks \#8 and \#9 in Table 5) had peculiar frequency characteristics that may influence the outcome when using different approaches to filtering the activity signal. A Fourier analysis of this chunk revealed a significant signal at 3.3 days. Pre-whitening of the data reveals two additional frequencies (Table 6) with frequencies near the rotational frequency, $\nu_{\text {rot }}$, and $\approx 5 \nu_{\text {rot }}$. The 3.3-day signal is of particular interest since this is uncomfortably close to the planet period. However, it is unlikely that this is due entirely to the planet since its amplitude is too large by a factor

of two. This signal is significant as a bootstrap analysis shows that FAP $=3.5 \times 10^{-4}$. In producing the residuals from Chunk8-9 that were used in LTF1 the 3.3-d period was kept in the data for the obvious reasons that it nearly coincides with the planetary signal. 
As a test we tried different filtering of the data only in Chunk8-9. The first and last half of the data in the chunk were filtered in different ways (e.g. second, third order polynomials, or sine functions). Different fits were performed after deleting points after a large time gap, first and last points from the data, data showing large variations with respect to adjacent values, etc. Ten different versions of filtering the chunk were tried and in all cases no more than 20 points were removed. The residuals were then added to the rest of LTF2 residuals. The Scargle power of the total residuals from all chunks ranged from as low as $z=7.0$ to as high as $z=11.4$. The average power was $8.8 \pm 1.3$. This corresponds to a range in FAP of $3-30 \%$. We also took the original LTF2 residuals from Chunk8-9 and replaced the corresponding time values in LTF1 and this alone boosted the Scargle power at the planet orbital frequency from $z=6.4(\mathrm{FAP}=0.4)$ to $z=9.75(\mathrm{FAP}=0.05)$.

In most cases the highest power in the periodogram in the frequency range $0.25 \mathrm{~d}^{-1}$ $<\nu<0.35 \mathrm{~d}^{-1}$ was not at the planet orbital frequency, but rather $P=2.94$ days $(\nu=$ $0.3397 \mathrm{~d}^{-1}$ ). The lower panel of Figure 13) shows one such filtered version of Chunk8-9 where the dominant peaks are not coincident with the planet orbital frequency.

We checked whether the planet signal could be detected in the subset RV measurements without data from Chunk8-9. Local trend filtering (LTF1) clearly shows no significant signal in this subset data (top left Figure 14). However, a 3.15-day periodic signal $\left(K=0.5 \mathrm{~m} \mathrm{~s}^{-1}\right)$ that was inserted into the data was found after applying LTF1 even without Chunk8-9. The same results were found when pre-whitening the RV data without Chunk8-9. A weak, but insignificant peak is found at the planet orbital frequency (top right panel Figure 14). Applying the pre-whitening to the data with the artificial planet $(P=3.15$ days $)$ can recover the input signal at a much higher significance (lower right panel in Figure 14). In summary, both pre-whitening and LTF methods should have been able to detect the planetary signal of $\alpha$ Cen $\mathrm{Bb}$ even without the data from Chunk8-9, but they do not. 
The behavior of the Scargle power as a function of the number of data points is a good way to assess the significance of a real periodic signal (see Hatzes \& Mkrtichian 2004). A real signal should have Scargle power that increases in an expected way as you add more data. The behavior of the statistical significance for the complete data was also inconsistent with the expectations of a real signal. Figure 15 shows the power at the orbital frequency of the planet as a function of the number of data points, $N$, using the residuals from local trend fitting and adding data sequentially in chronological order. We show the results for LTF1 and LTF2.

The figure also shows three simulated data sets. For these we added the orbit of $\alpha$ Cen $\mathrm{Bb}$ to the activity function generated from the sine components found by the pre-whitening of the individual epochs. The total RV curve was then sampled in the same way as the data and three different levels of random noise were added $\left(\sigma=0.8,1.0\right.$, and $\left.1.4 \mathrm{~m} \mathrm{~s}^{-1}\right)$. Local trend fitting was then applied using the same time windows as LTF1, but fitting the trends separately for each choice of random noise.

There are several features to note about this figure. The slope of the power versus $N$ function is much steeper for the simulated data, even with $\sigma$ as high as $1.4 \mathrm{~m} \mathrm{~s}^{-1}$. LTF1 shows power that is essentially flat except for the slight up-tick after the last data points are added. Even then the FAP is $\approx 40 \%$. The power from LTF2 behaves more erratically. There is a sharp increase as the first data points are used, followed by just as sharp a decline as more data are added. The "high" significance of the planet detection in LTF2 only occurs after adding the last 100 data points which is inconsistent with what one expects for random noise, This argues that the LTF1 choice of filtering may be a better approach to filtering out the activity variations.

We conclude that even though LTF2 shows modest power at the orbital frequency of the planet that this is not a significant detection and is consistent with the non-detection 
of LTF1.

\subsection{The Influence of Noise}

The question naturally arises: "Why do some approaches to removing the activity signal produce such discrepant results?" The sampling coupled with the noise characteristics may gives us some insight into this. In this case it is best to compare the unfiltered data in the Fourier domain. The amplitude spectrum of random noise with $\sigma=1.2 \mathrm{~m} \mathrm{~s}^{-1}$, our worse case estimate of the noise level, that is sampled in the same way as the data shows several peaks near the planet frequency and with amplitudes comparable the $K$-amplitude of the planet (top panel of Figure 16). Noise in the presence of the activity function shows an amplitude spectrum (middle panel of Figure 16) similar to that of the real data (lower panel of of Figure 16p. Spectral leakage from the activity signal into the frequency range $\nu \approx 0.3-0.31 \mathrm{~d}^{-1}$ may boost power in a noise peak coincident with the planet orbital frequency. The details as to how this noise peak is filtered may explain why the planet is present in some filtering approaches, but not others.

To explore further the influence of noise on the amplitude spectrum, synthetic data consisting of only random noise (no activity signal) with $\sigma=1.2 \mathrm{~m} \mathrm{~s}^{-1}$ were generated and sampled in the same way as the real data. A total of 100 random data sets were created using different seed values for the random number generator. The top panel of Figure 17 shows the distribution of the strongest peaks in the period range 2.85 days $<$ $P<3.8$ days $\left(0.26 \mathrm{~d}^{-1}<\nu<0.35 \mathrm{~d}^{-1}\right)$. This has a peak at $P=3.22 \pm 0.01$ days. The average amplitude of the peaks is $K=0.24 \pm 0.04$. In the unfiltered amplitude spectrum the velocity amplitude at the planet orbital frequency is $0.38 \mathrm{~m} \mathrm{~s}^{-1}$. The amplitude scales

approximately linear with $\sigma$, so for a noise level of $2 \mathrm{~m} \mathrm{~s}^{-1}$ the noise peaks would have an amplitude of $\approx 0.4 \mathrm{~m} \mathrm{~s}^{-1}$. 
The epoch subsets of the data show similar noise characteristics. As an example we only show the Epoch 3 data. The lower panel in Figure 17 show the noise peaks in the same frequency range for random data $\left(\sigma=1.2 \mathrm{~m} \mathrm{~s}^{-1}\right)$ sampled as the Epoch 3 data. The distribution is similar, but in this case the average peak amplitude is slightly higher at $K$ $=0.33 \pm 0.13 \mathrm{~m} \mathrm{~s}^{-1}$.

As seen from Figure 16 the activity signal may also boost the amplitude of the noise peak. The same simulation was performed including the activity function and random noise with $\sigma=1.2 \mathrm{~m} \mathrm{~s}^{-1}$. In this case the noise peaks had a mean amplitude of $K=0.39 \pm 0.04$, essentially the same value as in the unfiltered amplitude spectrum.

\section{Discussion}

Alpha Cen B is a modestly active star which shows RV activity jitter with an amplitude $\approx 1.5 \mathrm{~m} \mathrm{~s}^{-1}$ that is modulated with the 38-days rotation period of the star. Our ability to extract reliably planetary signals with a much smaller amplitude depends on how well this activity is filtered out and whether the filtering process introduces artifact frequencies. Two methods were used to eliminate the activity variations from the RV data of $\alpha$ Cen B: traditional Fourier pre-whitening and local trend filtering (LTF). By using different approaches to filter the data, we hoped to obtain a more accurate determination of the mass of $\alpha \mathrm{Cen} \mathrm{Bb}$ as well as to get a better assessment of the statistical significance of the detected signal.

The significance of the 3.24 days depends on how the activity variations are filtered. Table 7 summarizes the FAP of the planet signal as determined from the various filtering approaches applied in this paper. The values differ by factors of 100, from a modestly significant detection to a non-detection. Interestingly, the method that gives lowest FAP, 
pre-whitening of the full data set, is the one that has the poorest overall fit to the activity variations (see Figures 6-8). Pre-whitening of the individual epochs produces a better fit to the underlying activity (see Figure 3) and consequently the FAP increases by almost a factor of 20. Arguably the best fit to the activity signal, LTF1, produced no detection of the planet. The planet $\alpha$ Cen Bb seems to be elusive - for some filtering approaches it is there while for others it is not.

The pre-whitening of the full RV data and LTF2 produced results that were most consistent with the D2012 result. In the case of the pre-whitened we should assign little weight to the result as the fit to the overall activity variations is the poorest. There are some segments of the time series where the fit is good, others where it is significantly poorer than for other methods.

The results for LTF2 cannot be so summarily set aside. Here almost the same procedure of LTF1 was followed, with the exception of the trend fits to four time chunks. Ostensibly the fit to the underlying activity variations seems to as good as for LTF1. However, we have shown that the results depend sensitively on how the data of Chunk8-9 are filtered. This chunk is problematic because there are two large data gaps and the RV data shows complex variations. A Fourier analysis shows the presence of 3.3-d variations which is dangerously close to the planet period. Different approaches to fitting the activity variations in this chunk alone resulted in reduced power at the planet orbital frequency, and more importantly, resulted in higher power at a completely different frequency. Because of the complex time variations in this chunk, the filtering approach of LTF1 is probably better. Due to these difficulties the safest approach is to simply remove the data from Chunk8-9 from the analysis. Our simulations show that even without these measurements the planet should have been detected with a much higher statistical significance than was actually found for the data. 
One could argue that since some methods for filtering out the activity variations do find the planet signal that this qualifies as a confirmation of the presence of $\alpha$ Cen $\mathrm{Bb}$. However, a robust planet signal should be present at the same level regardless of how the data is analyzed. For a weak signal like $\alpha$ Cen $\mathrm{Bb}$ it is difficult to judge which method one should trust (harmonic analysis, pre-whitening, local trend fitting). We should emphasize that simulations have shown that if $\alpha$ Cen Bb were present according to the orbit of D2012 all methods (pre-whitening of full data set, pre-whitening of epoch data, and local trend filtering) should have detected the planet with high significance (FAP $<0.05 \%$ ).

The analysis in Section 3.5 points to noise as a possible explanation for the discrepant detections of $\alpha$ Cen Bb data. Simulations using random white noise with the "worse case" noise level of $1.2 \mathrm{~ms}^{-1}$ that were sampled like the data can create peaks in the amplitude spectrum near the period of the planet and with a comparable amplitude $(K=0.25-0.4$ $\mathrm{ms}^{-1}$ ). The amplitude of these noise peaks depends on two things: 1) the actual noise level of the RV data and its frequency spectrum, and 2) the underlying activity variations and its frequency spectrum.

The worse case noise level of $\sigma=1.2 \mathrm{~m} \mathrm{~s}^{-1}$ produces a noise amplitude of $K=0.24$ $\mathrm{ms}^{-1}$, but if the rms scatter is higher, the corresponding amplitude increases. However, this estimate of the noise level in the $\alpha$ Cen B RVs is derived after filtering out what we presumed were the activity variations. The unfiltered RV data has an rms scatter of 2.1 $\mathrm{ms}^{-1}$. If the true $\sigma$ is as high as $2 \mathrm{~ms}^{-1}$ the noise peak can have a value of $0.4 \mathrm{~m} \mathrm{~s}^{-1}$, comparable to the unfiltered $K$-amplitude of the planet in the Fourier spectrum.

The simulations also assumed Gaussian noise, but there are most likely systematic errors in the HARPS data and there is no guarantee that these also have a Gaussian distribution. Not only do we not know the true rms scatter of this systematic noise, but most importantly we have no knowledge of its frequency characteristics. Instead of being 
"white" the power spectrum of the systematic noise maybe "red" (i.e. overall slope rising to low frequencies), "blue" (slope that rises to higher frequencies), or with strong peaks (i.e. periodic signals in the data). Given that observations are made on nightly, weekly, monthly, and yearly timescales it is reasonable to expect that periodic structure is present in the Fourier amplitude spectrum of the systematic noise. We have seen that white noise can produce peaks at the right frequency and amplitude of the planet. We cannot be sure if systematic noise with a lower $\sigma$, but non-white frequency structure also boosts the amplitude of noise peaks in the Fourier spectrum at the frequency of interest. Perhaps LTF1 is a better way of filtering noise in the presence of the data window which is why it produces no significant power at the planet orbital frequency.

The activity variations can also boost the amplitude of noise peaks. Using our simple activity function and random noise with $\sigma=1.2 \mathrm{~m} \mathrm{~s}^{-1}$ we were able to produce noise peaks with amplitudes consistent with the planet velocity $K$-amplitude. The frequency spectrum of the activity signal for $\alpha$ Cen B is certainly complex, one that results from periodic and semi-periodic variations. Intrinsic stellar variations which can be stochastic (e.g. granulation, spot evolution, etc.) introduce "noise" with their own frequency structure and this only complicates matters further. The activity signal, coupled with noise and the sampling window may also produce spurious peaks in the amplitude spectrum that may not be filtered out appropriately. By lowering the surrounding Fourier noise floor the filtering process may only make these spurious peaks look more significant than they really are. For the detection of weak signals due to planets it is essential to use different ways of filtering the data to ensure that we arrive at consistent results. 


\section{Conclusions}

This investigation into the RV variations of $\alpha$ Cen B using different approaches to filtering out the activity signal was not able to confirm the presence of the Earth-mass planet in a 3.24 day orbit. The detected "planet" seemed to be highly sensitive to the details in how the activity variations are removed. Alpha Cen Bb should have been detected by all methods that were employed and at the same level of significance. A possible explanation for the planet signal found by D2012 $\alpha$ Cen Bb is that it is a noise peak in the data whose statistical power has been boosted by a combination of the frequency characteristics of the noise, the under-sampling of the activity signal, and the filtering process. This work cannot prove unequivocally that the RV signal attributed to $\alpha \mathrm{Cen} \mathrm{Bb}$ is in fact noise. More analyses are needed, and most importantly more data taken should be taken with higher cadence so as to sample adequately the activity variations. Only when the signal of $\alpha$ Cen $\mathrm{Bb}$ rises with certainty above the noise level will we be certain of this planet.

In detecting the RV variations of low-mass exoplanets in the presence of activity noise it is essential to confirm the detection using different filtering approaches. We have shown that standard Fourier pre-whitening can be a useful tool for finding such signals, but the result should be verified using a different approach that is tailored to detecting the frequency of interest. It should not be used indiscriminately.

Finally, a formal low false alarm probability is no guarantee that a periodic signal in RV data is in fact significant, particularly when one has modified the data through a filtering process. Simulated data should be used to ensure that the signal was detected at the proper level and that its significance behaves in the expected manner given the best estimate of the noise characteristics of the data. Quoted low values of the FAP for activity filtered data should be treated with caution.

On a positive note, our analysis also shows that it is possible to extract the RV signal 
of a short-period Earth-mass planet in the presence or activity noise given exquisite quality data such as those taken with HARPS. However, high cadence observations are required.

\section{Acknowledgments}

The author wishes to thank Bill Cochran, Mike Endl, and Guenther Wuchterl for useful comments on the manuscript. We also thank the anonymous referee for her/his careful review and suggestions. This resulted in a much improved manuscript. 


\section{REFERENCES}

Bakos, G.A., Csubry, Z. Penev, K. et al. 2013, PASP, 125, 154

Boisse, I., Bouchy, F., Hébrard, G., Bonfils, X., Santos, N., Vauclair, S. 2011, A\&A, 528, 4

Dumusque, X., Pepe, F., Lovis, C. et al. 2012, Nature, 491, 207 (D2012)

Ferraz-Mello, S., Tadeu Dos Santos, M., Beaugé C., Michtchenko, T.A., Rodriguez, A. 2011, A\&A, 531, 161

García Hernández, A. et al. 2009, A\&A, 506, 79

Grziwa, S., Pätzold, M., Carone, L. 2012, MNRAS, 4201045

Hatzes, A.P. AN, 2002, 323, 392

Hatzes, A.P., Dvorak, R., Wuchterl, G. et al. 2010, A\&A, 520, 93

Hatzes, A.P., Fridlund, M., Nachmani, G. et al. 2011, A\&A, 743, 75

Hatzes, A.P., Mkrtichian, D. 2004, MNRAS, 351, 663

Hatzes, A.P. Nature, 2012, 491, 200

Kovács, G., Bakos, G., Noyes, R.W. 2005, MNRAS, 356, 557

Kuschnig, R., Weiss, W.W., Gruber, R., Bely, P.Y., Jenkner, H. 1997, 328, 544

Léger, A., Rouan, D., Schneider, J. et al. 2009, A\&A, 506, 287

Lenz, P., \& Breger, M. 2004, The A-Star Puzzle, 224, 786

Mayor, M., Udry, S., Lovis, C., et al. 2009, A\&A, 493, 639

Pepe, F., Lovis, C., Ségransan, D. et al. 2011, A\&A, 534, 58 
Queloz, D., Bouchy, D., Moutou, C. et al. 2009, A\&A, 506, 303

Saar, S.H., Donahue, R.A. 1997, ApJ, 485, 319

Scargle, J.D. 1982, ApJ, 263, 835

Tuomi, M., Anglada-Escudé, G., Gerlach, E., Jones, H.R.A., Reinters, A., Rivera, E.J. Vogt, S.S., Butler, R.P. 2013, A\&A, 549, 48 
Table 1: Epochs of the HARPS data

\begin{tabular}{cllcc}
\hline \hline & $\begin{array}{l}\text { Dates } \\
\text { Epoch }\end{array}$ & $\begin{array}{l}\Delta \mathrm{T} \\
\text { JD-2400000 }\end{array}$ & $\begin{array}{c}N_{\text {obs }} \\
\text { (days) }\end{array}$ & $\begin{array}{c}\sigma \\
\left(\mathrm{m} \mathrm{s}^{-1}\right)\end{array}$ \\
\hline 1 & $54524.9-54648.6$ & 124 & 42 & 1.15 \\
2 & $54878.8-55048.6$ & 169 & 243 & 1.86 \\
3 & $54278.7-55359.5$ & 91 & 120 & 2.42 \\
4 & $55611.8-55755.5$ & 144 & 154 & 2.20 \\
\hline
\end{tabular}

Table 2: Pre-whitening results for the RV data

\begin{tabular}{ccccc}
\hline $\mathrm{N}$ & $\begin{array}{c}\text { Frequency } \\
\left(\mathrm{d}^{-1}\right)\end{array}$ & $\begin{array}{c}\text { Period } \\
\text { (days })\end{array}$ & $\begin{array}{c}K \text {-amplitude } \\
\left(\mathrm{m} \mathrm{s}^{-1}\right)\end{array}$ & Phase \\
\hline \hline $\mathrm{f}_{1}$ & $0.02558 \pm 0.00002$ & $39.09 \pm 0.03$ & $1.89 \pm 0.08$ & $0.00 \pm 0.01$ \\
$\mathrm{f}_{2}$ & $0.00131 \pm 0.00005$ & $763.36 \pm 29.12$ & $0.69 \pm 0.08$ & $0.79 \pm 0.01$ \\
$\mathrm{f}_{3}$ & $0.08163 \pm 0.00004$ & $12.25 \pm 0.006$ & $1.00 \pm 0.08$ & $0.49 \pm 0.01$ \\
$\mathrm{f}_{4}$ & $0.10438 \pm 0.00005$ & $9.58 \pm 0.005$ & $0.75 \pm 0.08$ & $0.13 \pm 0.02$ \\
$\mathrm{f}_{5}$ & $0.00603 \pm 0.00004$ & $165.83 \pm 1.10$ & $0.97 \pm 0.08$ & $0.78 \pm 0.02$ \\
$\mathrm{f}_{6}$ & $0.06633 \pm 0.00005$ & $15.79 \pm 0.01$ & $0.71 \pm 0.08$ & $0.57 \pm 0.02$ \\
$\mathrm{f}_{7}$ & $0.03321 \pm 0.00005$ & $101.11 \pm 0.15$ & $0.67 \pm 0.09$ & $0.62 \pm 0.02$ \\
$\mathrm{f}_{8}$ & $0.07841 \pm 0.00005$ & $12.75 \pm 0.047$ & $0.77 \pm 0.08$ & $0.05 \pm 0.03$ \\
$\mathrm{f}_{9}$ & $0.30906 \pm 0.00009$ & $3.2356 \pm 0.0001$ & $0.40 \pm 0.08$ & $0.33 \pm 0.03$ \\
\hline
\end{tabular}


Table 3: Tests of the Fourier procedure on the full data set

\begin{tabular}{ccccc}
\hline \hline $\begin{array}{c}P_{\text {in }} \\
(\text { days })\end{array}$ & $\begin{array}{c}K_{\text {in }} \\
\left(\mathrm{m} \mathrm{s}^{-1}\right)\end{array}$ & $\begin{array}{c}P_{\text {out }} \\
(\text { days })\end{array}$ & $\begin{array}{c}K_{\text {out }} \\
\left(\mathrm{m} \mathrm{s}^{-1}\right)\end{array}$ & FAP \\
\hline 3.350 & 0.50 & $3.349 \pm 0.001$ & $0.54 \pm 0.08$ & $<5.0 \times 10^{-5}$ \\
3.300 & 0.50 & $3.299 \pm 0.001$ & $0.64 \pm 0.09$ & $<5.0 \times 10^{-5}$ \\
3.236 & 0.50 & $3.236 \pm 0.001$ & $0.52 \pm 0.08$ & $1.5 \times 10^{-5}$ \\
3.200 & 0.50 & $3.203 \pm 0.001$ & $0.54 \pm 0.10$ & $2.0 \times 10^{-5}$ \\
3.150 & 0.50 & $3.151 \pm 0.001$ & $0.58 \pm 0.10$ & $<5.0 \times 10^{-5}$ \\
\hline
\end{tabular}


Table 4: Pre-whitening results for the Epoch data

\begin{tabular}{ccccc}
\hline \hline ID & $\begin{array}{c}\text { Frequency } \\
\left(\mathrm{d}^{-1}\right)\end{array}$ & $\begin{array}{c}K \text {-amplitude } \\
\left(\mathrm{m} \mathrm{s}^{-1}\right)\end{array}$ & Phase & Comment \\
\hline$f_{11}$ & $0.020 \pm 0.001$ & $0.89 \pm 0.29$ & $0.91 \pm 0.04$ & Epoch 1 \\
\hline$f_{21}$ & $0.0043 \pm 0.001$ & $2.01 \pm 0.15$ & $0.27 \pm 0.02$ & Epoch 2 \\
$f_{22}$ & $0.0692 \pm 0.001$ & $0.76 \pm 0.15$ & $0.82 \pm 0.03$ & Epoch 2 \\
$f_{23}$ & $0.1347 \pm 0.001$ & $0.71 \pm 0.15$ & $0.07 \pm 0.03$ & Epoch 2 \\
\hline$f_{31}$ & $0.0104 \pm 0.0002$ & $1.73 \pm 0.16$ & $0.33 \pm 0.02$ & Epoch 3 \\
$f_{32}$ & $0.0284 \pm 0.0003$ & $2.52 \pm 0.16$ & $0.88 \pm 0.02$ & Epoch 3 \\
$f_{33}$ & $0.0644 \pm 0.0016$ & $0.76 \pm 0.16$ & $0.38 \pm 0.04$ & Epoch 3 \\
$f_{34}$ & $0.1510 \pm 0.0016$ & $0.69 \pm 0.16$ & $0.60 \pm 0.04$ & Epoch 3 \\
\hline$f_{41}$ & $0.0267 \pm 0.0005$ & $1.59 \pm 0.15$ & $0.21 \pm 0.02$ & Epoch 4 \\
$f_{42}$ & $0.0818 \pm 0.0005$ & $1.63 \pm 0.13$ & $0.65 \pm 0.02$ & Epoch 4 \\
$f_{43}$ & $0.1028 \pm 0.0014$ & $1.63 \pm 0.14$ & $0.73 \pm 0.04$ & Epoch 4 \\
\hline
\end{tabular}


Table 5: Time chunks used for the Local Trend Filtering

\begin{tabular}{|c|c|c|c|c|c|c|}
\hline Chunk \# & $\begin{array}{c}\text { Dates } \\
(\mathrm{JD}-2400000)\end{array}$ & $\begin{array}{c}\Delta \mathrm{T} \\
(\text { days })\end{array}$ & $N_{\text {orb }}$ & $N_{\text {data }}$ & Fitting Function & $\begin{array}{c}\sigma \\
\left(\mathrm{m} \mathrm{s}^{-1}\right)\end{array}$ \\
\hline 1 & $54524.91-54530.83$ & 5.93 & 1.83 & 7 & constant & 0.53 \\
\hline 2 & $54548.82-54557.76$ & 8.94 & 2.16 & 10 & linear & 0.57 \\
\hline 3 & $54562.78-54571.72$ & 8.90 & 2.75 & 9 & linear & 1.19 \\
\hline 4 & $54610.72-54617.62$ & 6.88 & 2.13 & 5 & constant & 1.13 \\
\hline 5 & $54638.69-54648.64$ & 9.95 & 2.99 & 11 & constant & 1.06 \\
\hline 6 & $54878.80-54884.89$ & 6.09 & 1.86 & 10 & polynomial $n=2$ & 0.39 \\
\hline 7 & $54913.77-54920.90$ & 7.73 & 3.01 & 21 & polynomial $n=2$ & 0.55 \\
\hline 8 & $54933.70-54938.82$ & 5.12 & 1.58 & 18 & polynomial $n=2$ & 0.78 \\
\hline 9 & $54946.67-54956.82$ & 10.15 & 3.14 & 30 & polynomial $n=3$ & 0.78 \\
\hline 10 & $54988.53-55002.68$ & 14.15 & 4.34 & 29 & linear & 0.84 \\
\hline 11 & $55036.55-55048.58$ & 12.04 & 3.72 & 18 & linear & 1.10 \\
\hline 12 & $55278.74-55301.88$ & 23.14 & 7.15 & 62 & polynomial $n=3$ & 0.90 \\
\hline 13 & $55321.60-55328.77$ & 7.17 & 2.20 & 21 & polynomial $n=2$ & 0.84 \\
\hline 14 & $55334.59-55342.76$ & 8.17 & 2.52 & 19 & polynomial $n=2$ & 1.07 \\
\hline 15 & $55350.66-55355.59$ & 4.93 & 1.52 & 11 & polynomial $n=2$ & 0.95 \\
\hline 16 & $55611.79-55616.80$ & 5.00 & 1.23 & 9 & constant & 0.80 \\
\hline 18 & $55619.77-55648.84$ & 29.07 & 8.98 & 44 & polynomial $n=2$ & 0.82 \\
\hline 19 & $55656.67-55663.81$ & 7.14 & 2.21 & 37 & polynomial $n=2$ & 1.04 \\
\hline 20 & $55672.65-55692.70$ & 9.95 & 3.08 & 36 & multi-sine & 1.32 \\
\hline 21 & $55711.57-55728.53$ & 16.95 & 5.24 & 11 & linear & 1.58 \\
\hline
\end{tabular}


Table 6: Pre-whitening results for Chunk8-9

\begin{tabular}{cccc}
\hline \hline $\begin{array}{c}\text { Frequency } \\
\left(\mathrm{d}^{-1}\right)\end{array}$ & $\begin{array}{c}\text { Period } \\
(\text { days })\end{array}$ & $\begin{array}{c}K \text {-amplitude } \\
\left(\mathrm{m} \mathrm{s}^{-1}\right)\end{array}$ & Phase \\
\hline $0.3020 \pm 0.0034$ & $3.31 \pm 0.03$ & $0.963 \pm 0.15$ & $0.77 \pm 0.02$ \\
$0.1321 \pm 0.0036$ & $7.57 \pm 0.21$ & $0.902 \pm 0.15$ & $0.68 \pm 0.02$ \\
$0.0271 \pm 0.0070$ & $36.90 \pm 9.56$ & $0.463 \pm 0.15$ & $0.64 \pm 0.05$ \\
\hline
\end{tabular}


Table 7: FAP values for the planet signal

\begin{tabular}{lc}
\hline \hline Filter & FAP \\
\hline Full data Pre-whitening & 0.004 \\
Epoch Pre-whitening & 0.07 \\
Local Trend fitting (LFT1) & 0.40 \\
Local Trend fitting (LFT2) & 0.005 \\
LFT2: various filters to Chunk8-9 & $0.03-0.3$ \\
\hline
\end{tabular}




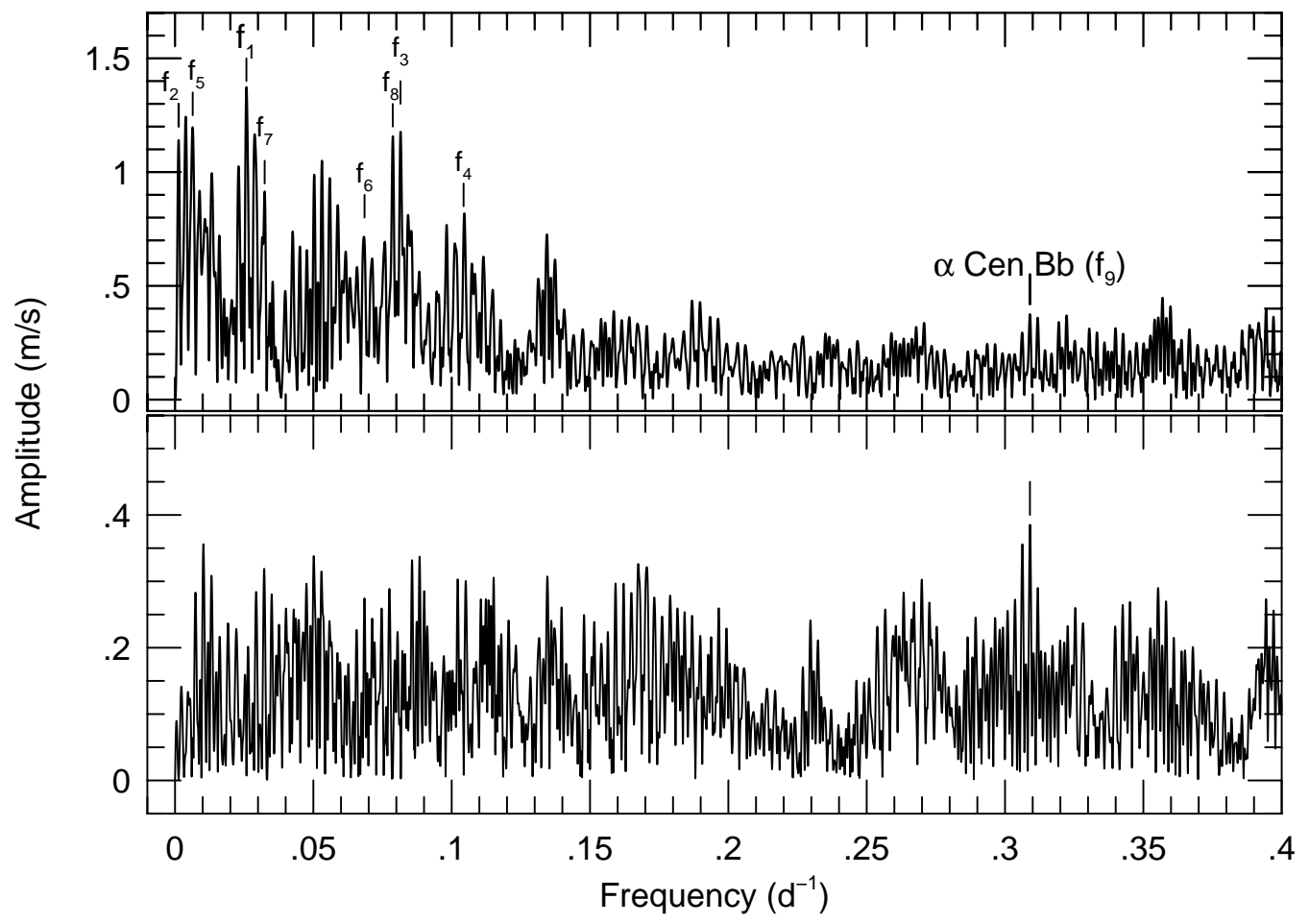

Fig. 1.- (Top) The DFT amplitude spectrum of the full RV data. The marked frequencies indicate those found and removed by the pre-whitening process (Table 2). The orbital frequency of $\alpha$ Cen $\mathrm{Bb}$ ( $f_{9}$ from Table 2) is also shown. (Bottom) The final pre-whitened amplitude spectrum with only $f_{9}$ remaining. 


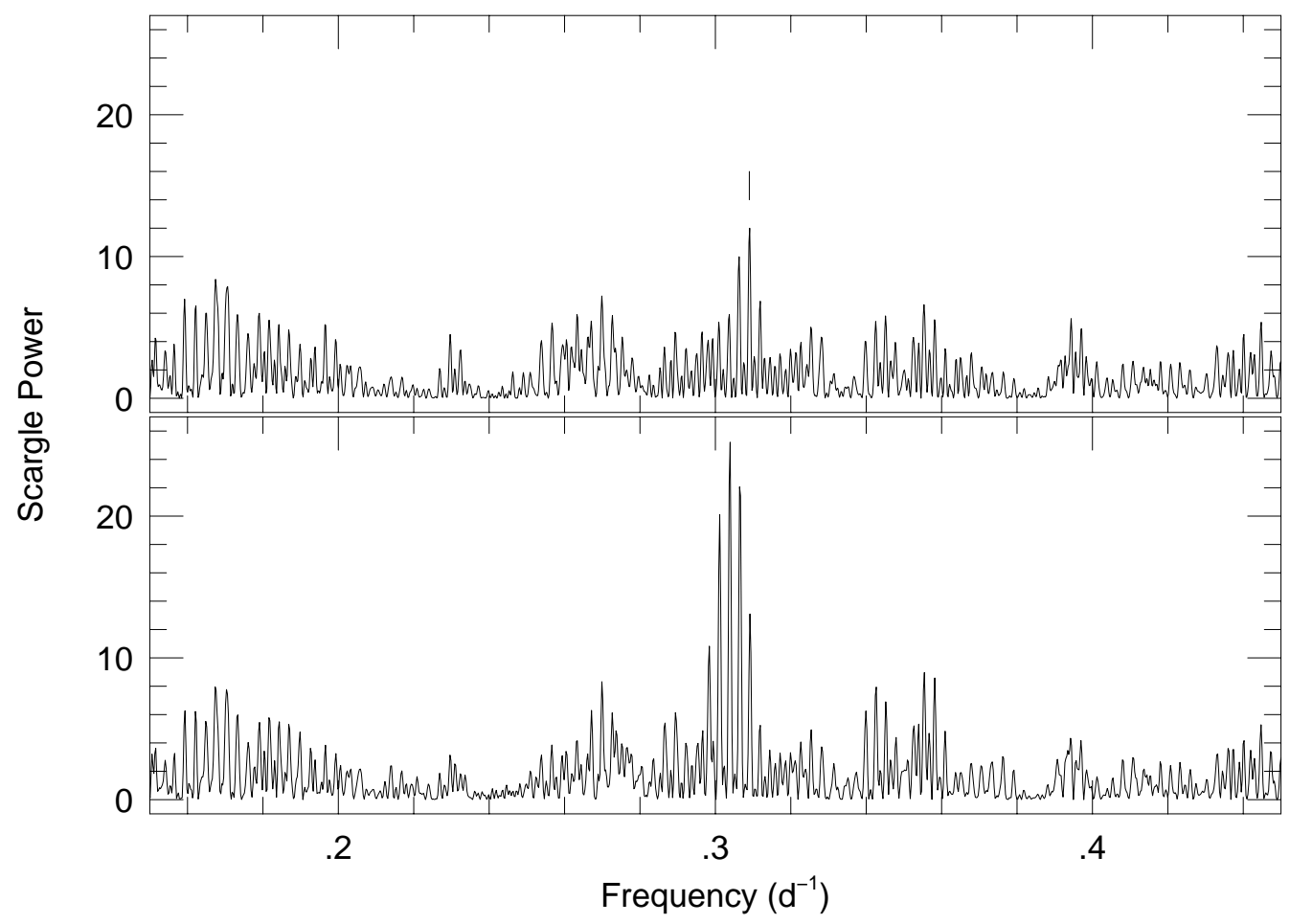

Fig. 2.- (Top) The Scargle periodogram of the residual RVs of $\alpha$ Cen after removing the first eight frequencies in Table 1. The vertical line marks the orbital frequency of $\alpha$ Cen Bb. (Bottom) The periodogram of the RV residuals with a simulated planet signal $(\nu=$ $0.303 \mathrm{~d}^{-1}=3.29$ days $K=0.5 \mathrm{~m} \mathrm{~s}^{-1}$ ) inserted into the data prior to pre-whitening. 


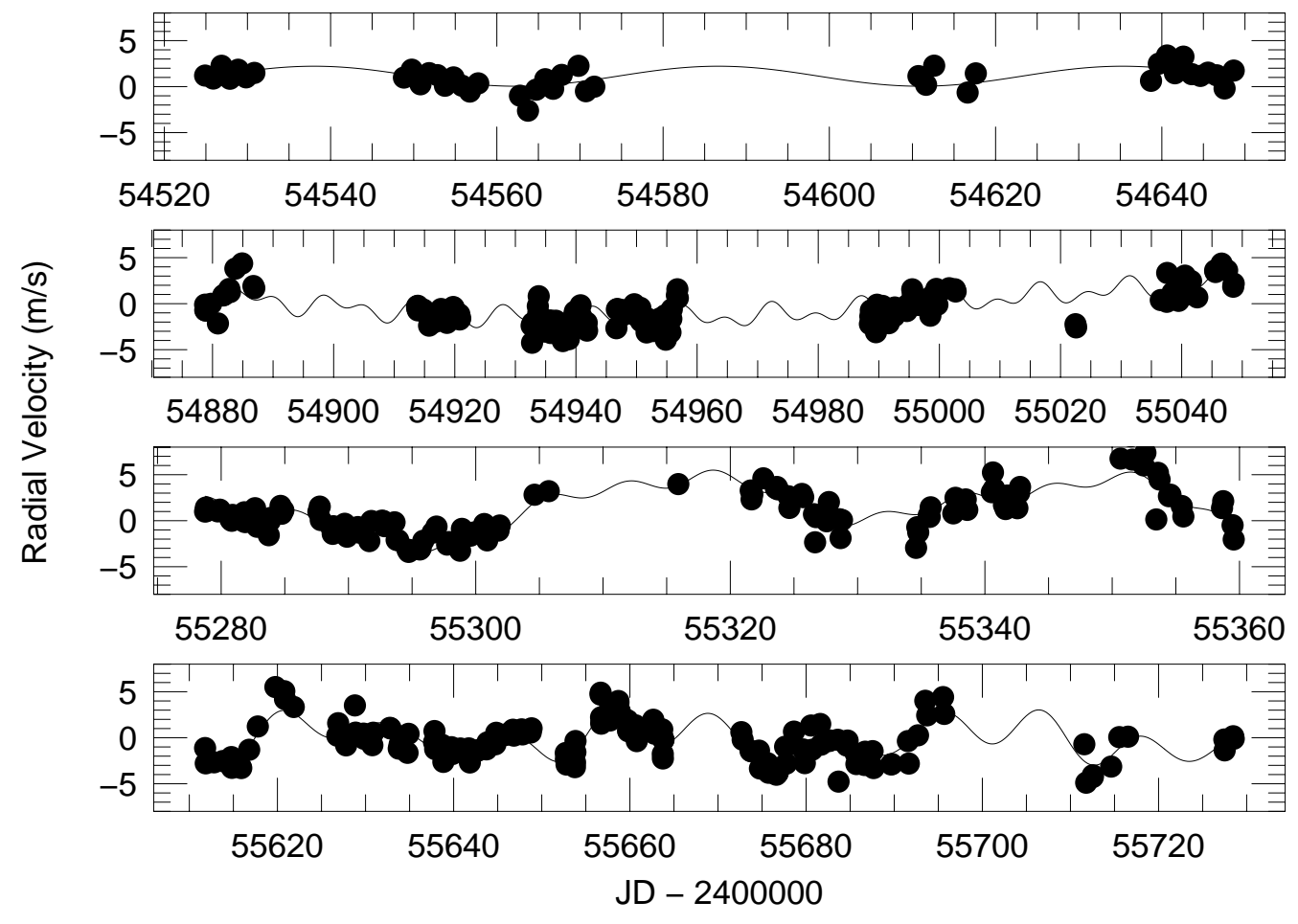

Fig. 3.- The underlying activity signal for the four epochs computed using the pre-whitening process separately for each epoch. 


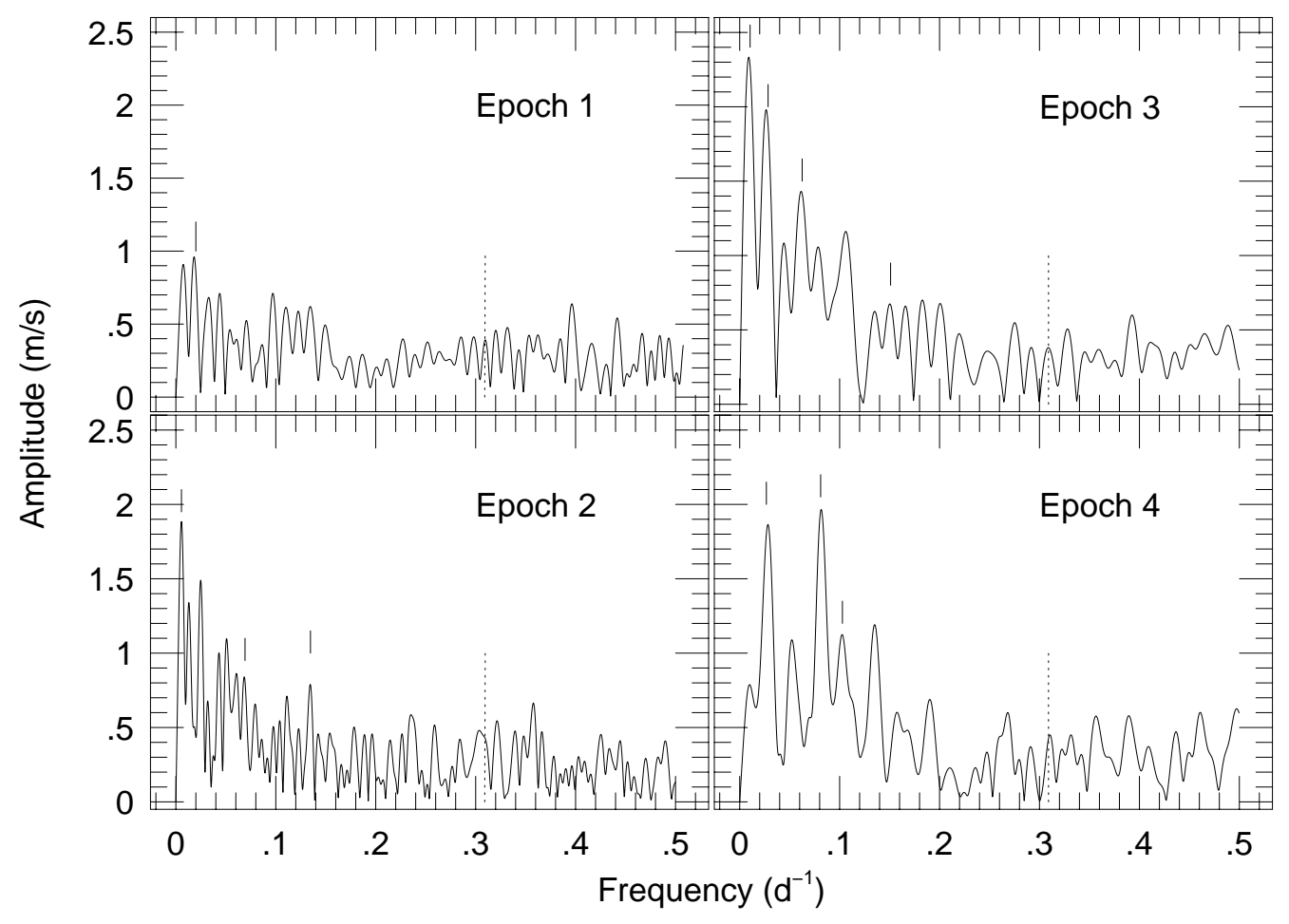

Fig. 4.- The DFT for the four epochs of RV measurements. The vertical lines mark the frequencies removed via the pre-whitening process and the dashed vertical line is the planet orbital frequency. 


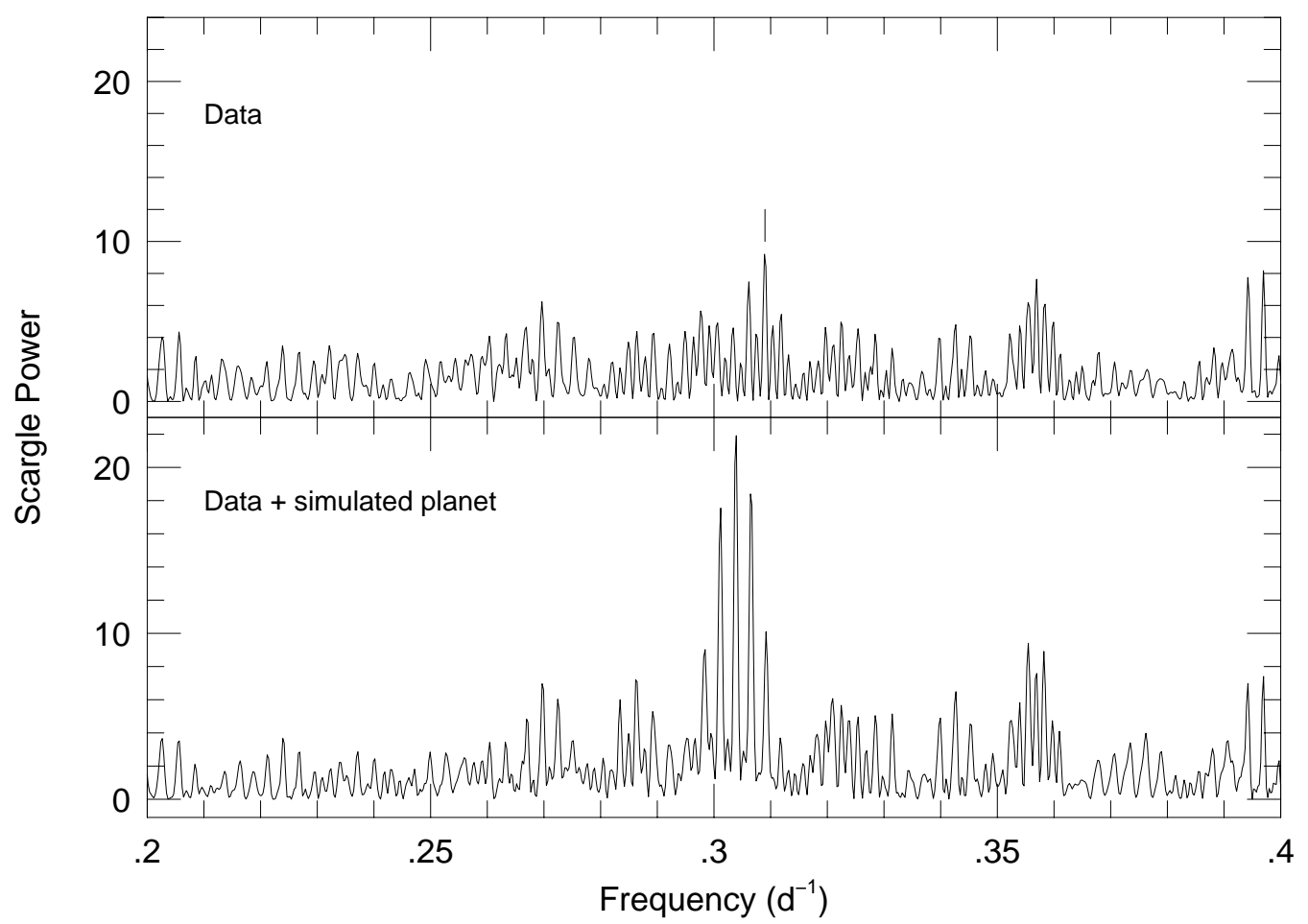

Fig. 5.- (Top) The Scargle periodogram of the pre-whitened epoch RV measurements for $\alpha$ Cen B. The vertical line marks the orbital frequency of $\alpha$ Cen Bb. (Bottom) The Scargle periodogram of the pre-whitened epoch RV measurements for $\alpha$ Cen B but with the addition of a simulated planet having $P=3.29 \mathrm{~d}\left(\nu=0.303 \mathrm{~d}^{-1}\right)$ and $K=0.5 \mathrm{~m} \mathrm{~s}^{-1}$. 


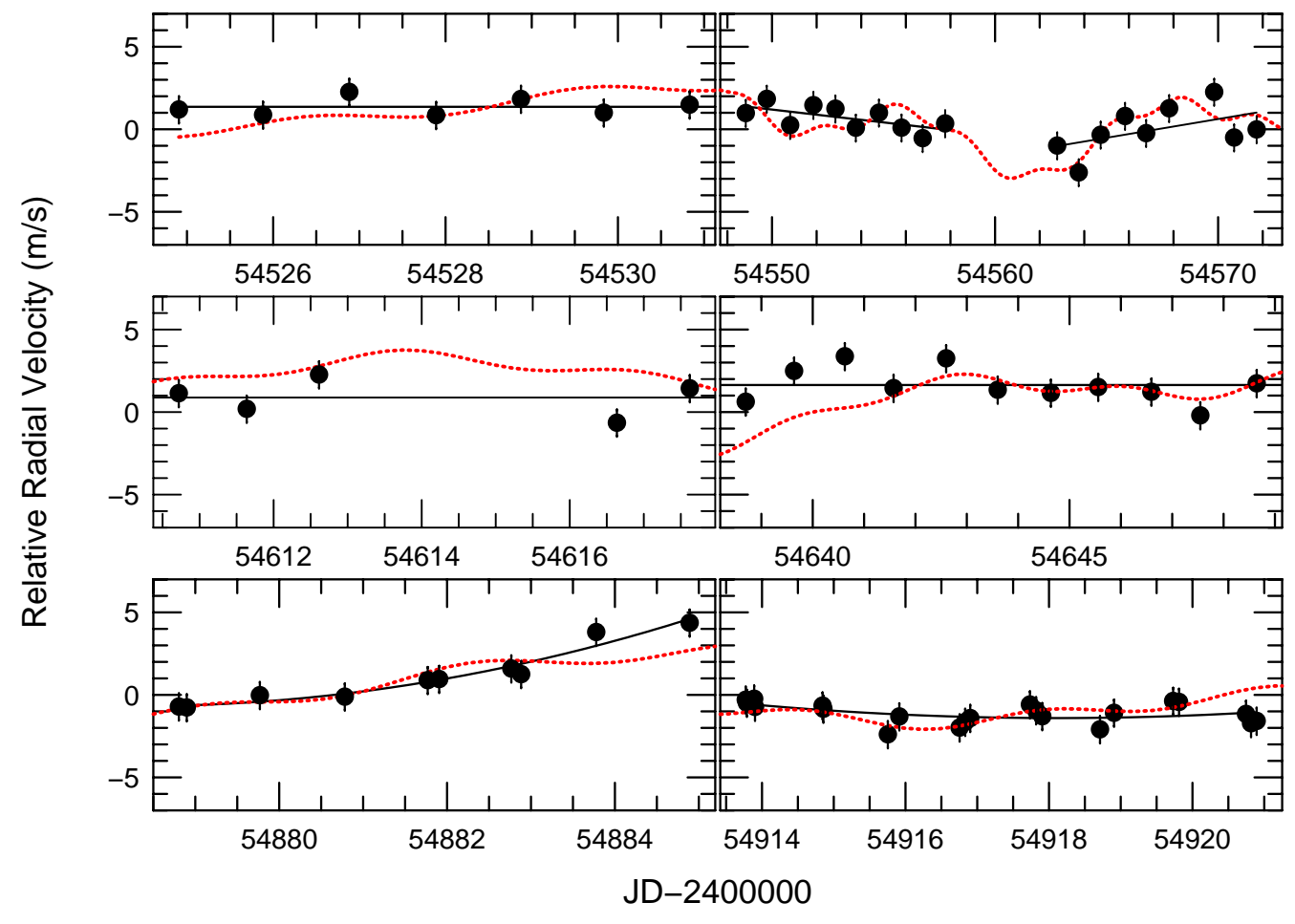

Fig. 6. - The first six time chunks used for the local trend fitting of the activity signal. The solid line represents the local fit to the trend. The dotted red line is the fit to the activity using the pre-whitening process on the full data set (Table 2 but without the planet signal). 


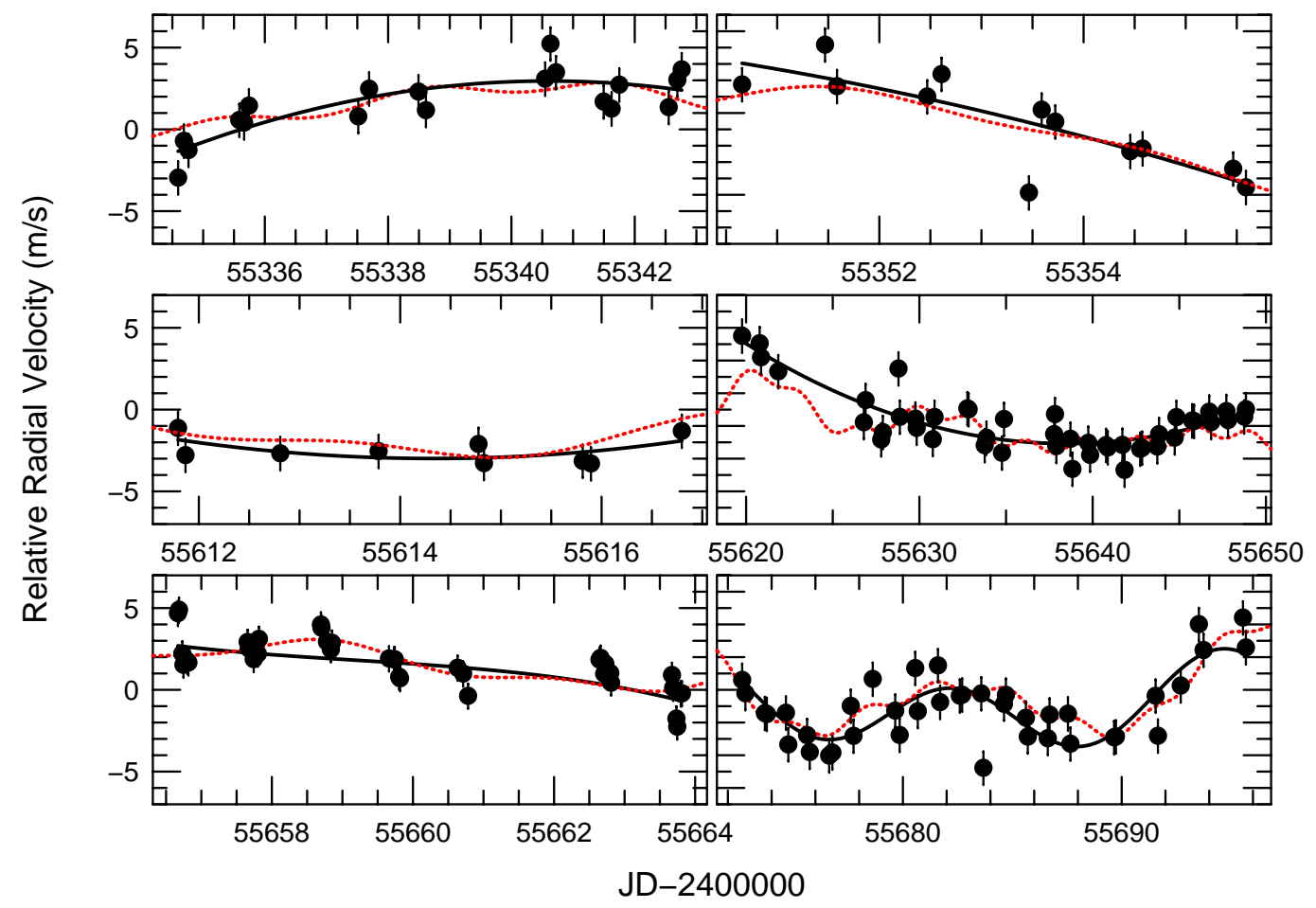

Fig. 7.- Same as for Fig. 6, but for the next six time chunks. 


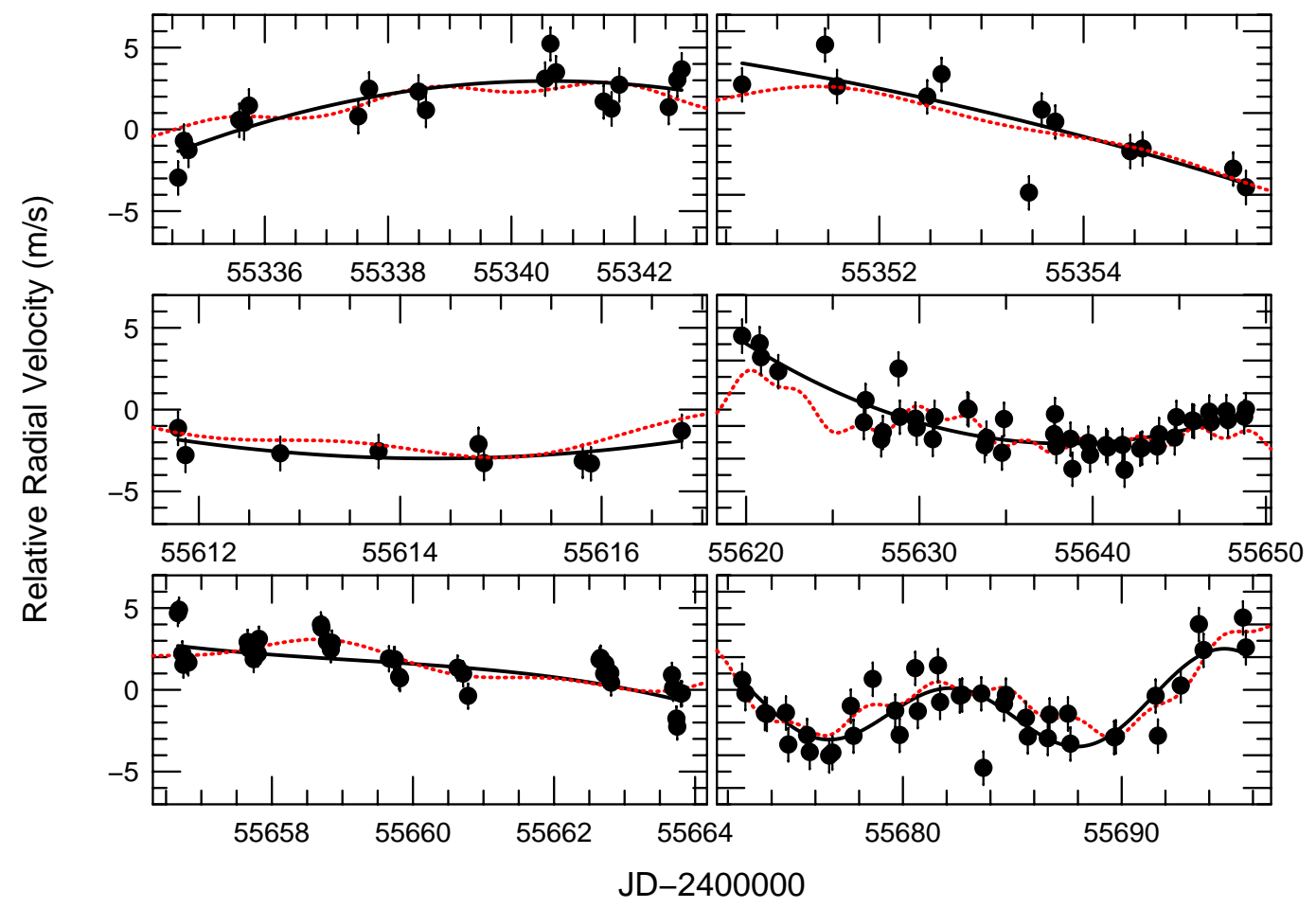

Fig. 8. - The next six time chunks used for local trend fitting. 


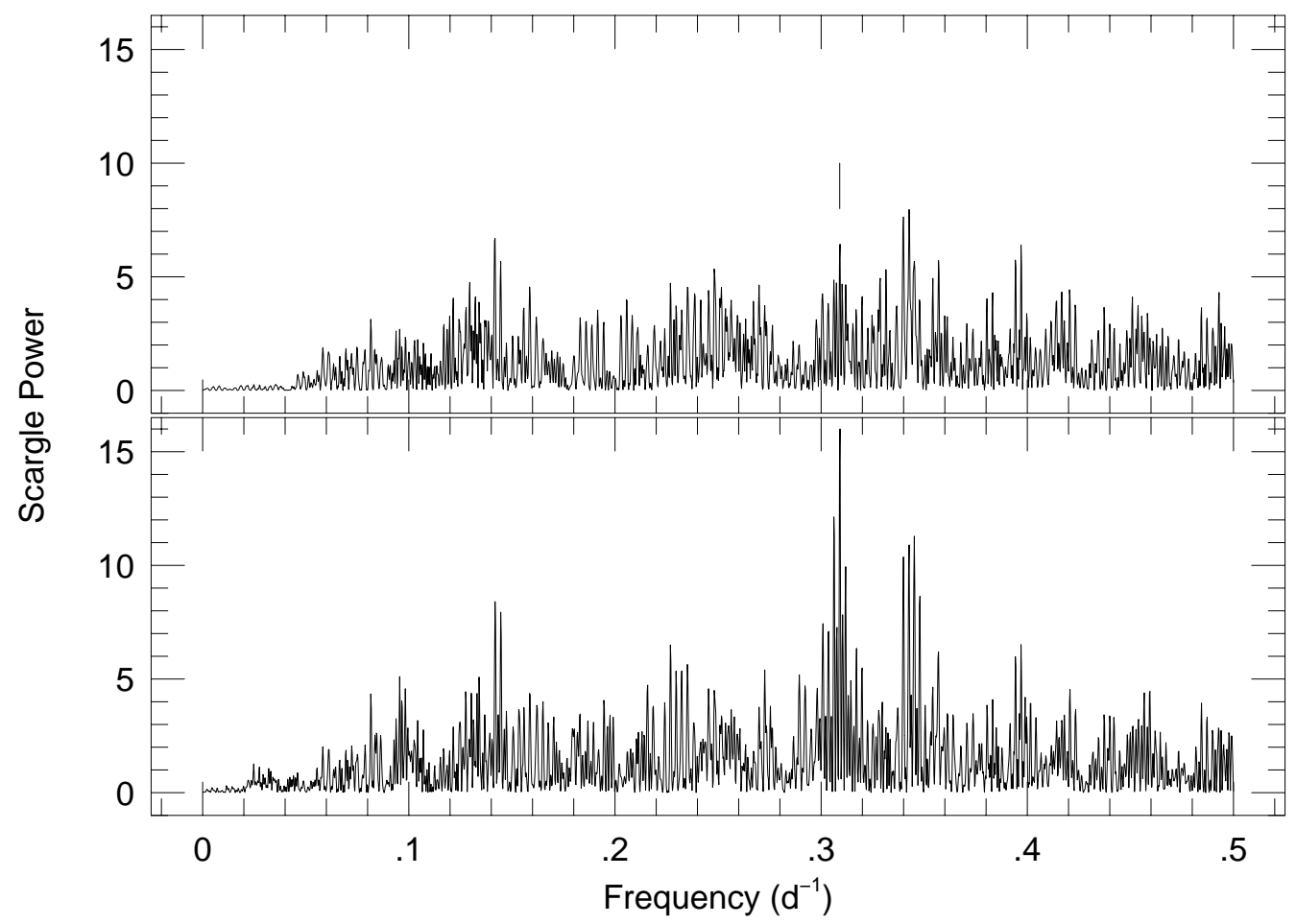

Fig. 9. - (Top) The periodogram of the RV residuals after removing the local trends shown in Figures 648 (LTF1). (Bottom) Periodogram of the local trend fitted RV residuals using the RV data after removing the sine component at the planet orbital frequency found by pre-whitening ( $f_{9}$ in Table 2$)$ and inserting the orbit of $\alpha$ Cen $\mathrm{Bb}(P=3.24$ days, $K=0.51$ $\left.\mathrm{ms}^{-1}\right)$. 


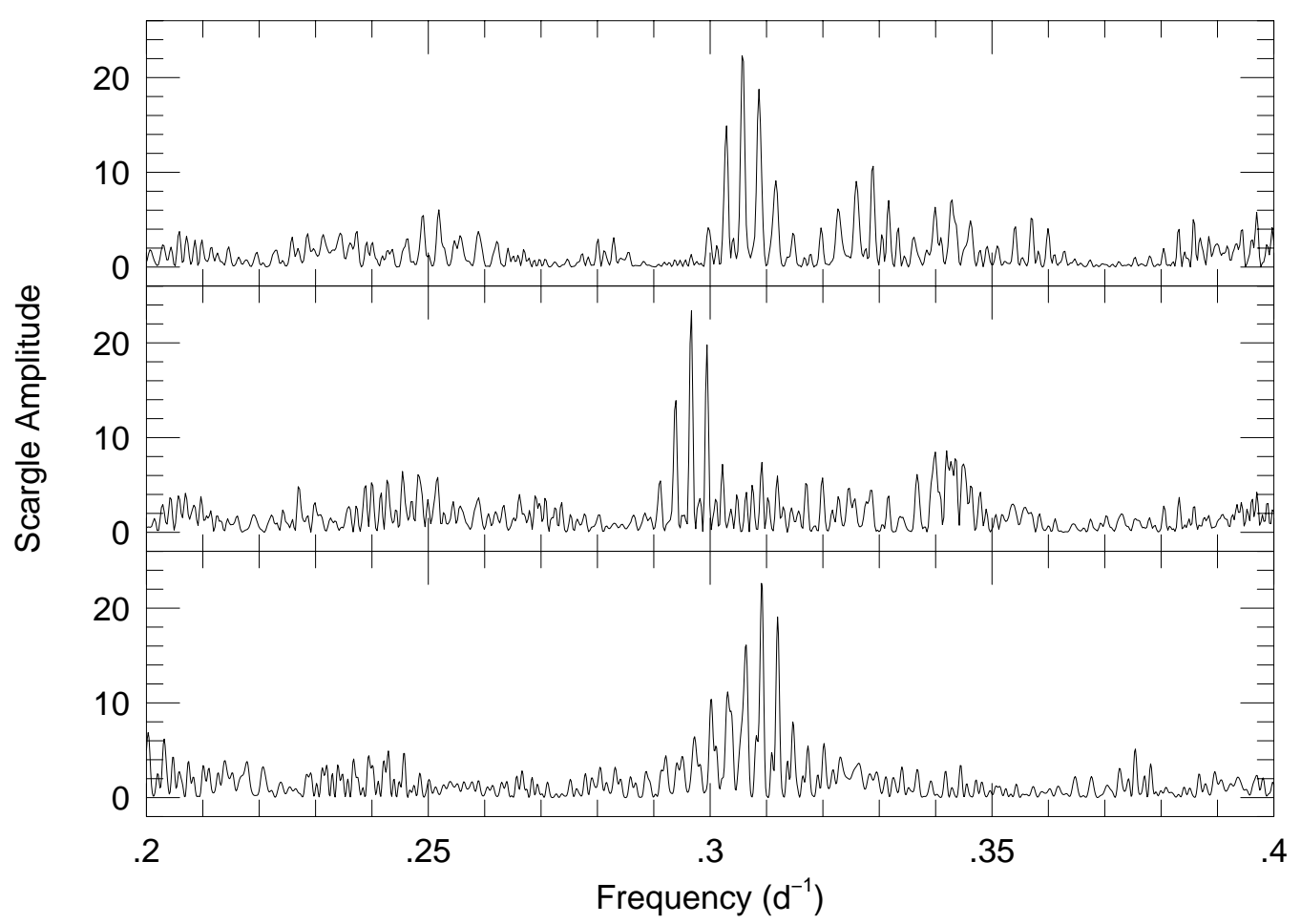

Fig. 10.- Periodograms of simulated data after applying local trend fitting. (Top) Simulation using the actual RV data where the planetary signal was removed and an artificial signal with $P=3.27$ days, $K=0.5 \mathrm{~m} \mathrm{~s}^{-1}$ added back into the data before pre-whitening. (middle) Simulation using the real RV data and a simulated signal with $P=3.37 \mathrm{~d}, K=$ $0.5 \mathrm{~m} \mathrm{~s}^{-1}$ added. (Bottom) Simulated data using an activity signal consisting of a multi-sine fit generated with the first eight frequencies in Table 2 . This activity signal has the same temporal sampling as the data and random noise at a level of $0.8 \mathrm{~m} \mathrm{~s}^{-1}$. A planet signal with $P=3.24$ days and $K=0.5 \mathrm{~m} \mathrm{~s}^{-1}$ was also added to the data. In all cases the input signal was recovered at high statistical significant. 


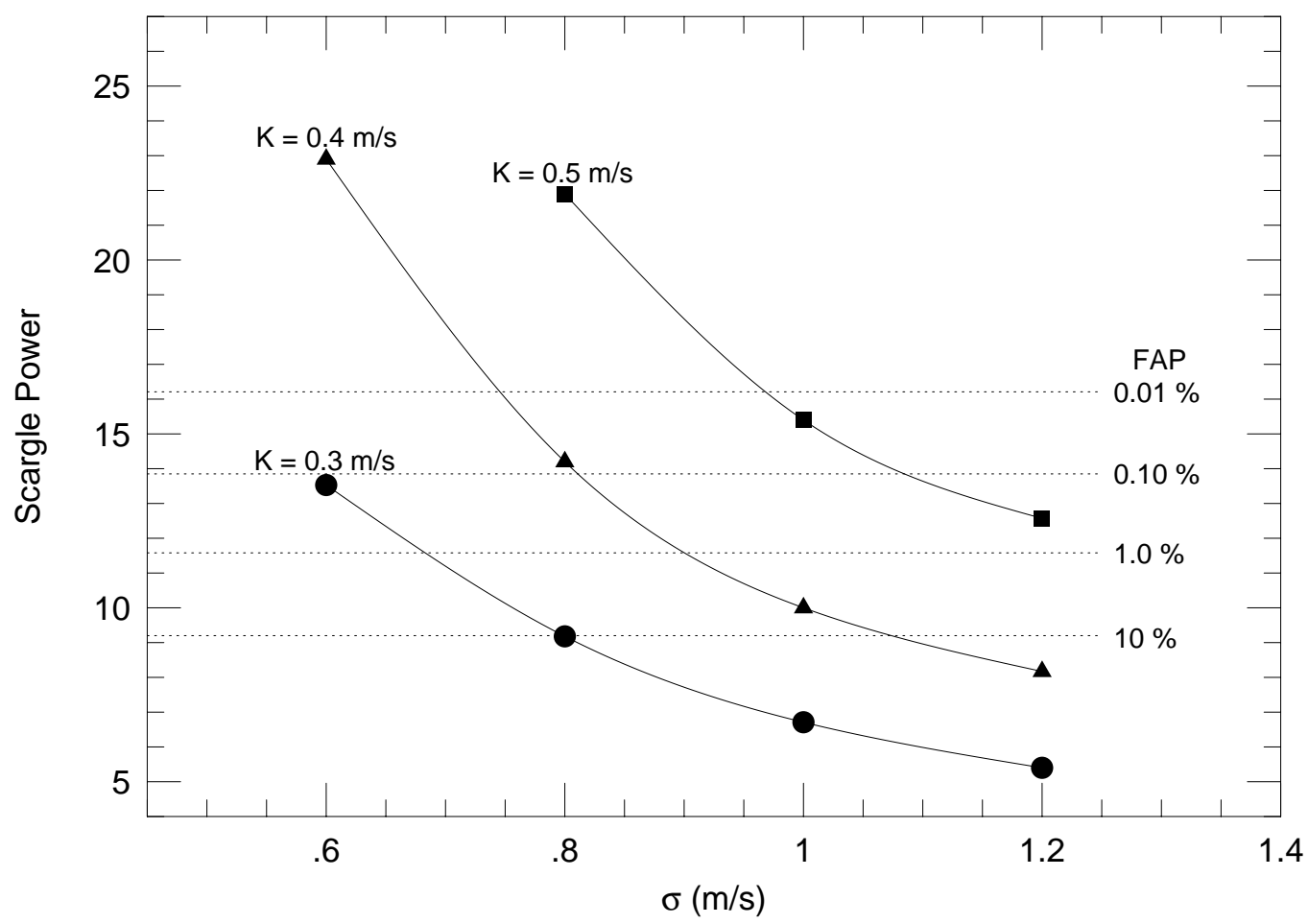

Fig. 11. - The detected $K$-amplitude for a 3.24-day period planet in the HARPS data as a function of the rms scatter, $\sigma$, of the data. This is based on simulated data using the multisine component model for the activity and the orbital parameters from D2012, but with different $K$-values taken from D2012. The ordinate is in Scargle power and the horizontal lines show the corresponding FAP determined via a bootstrap. 


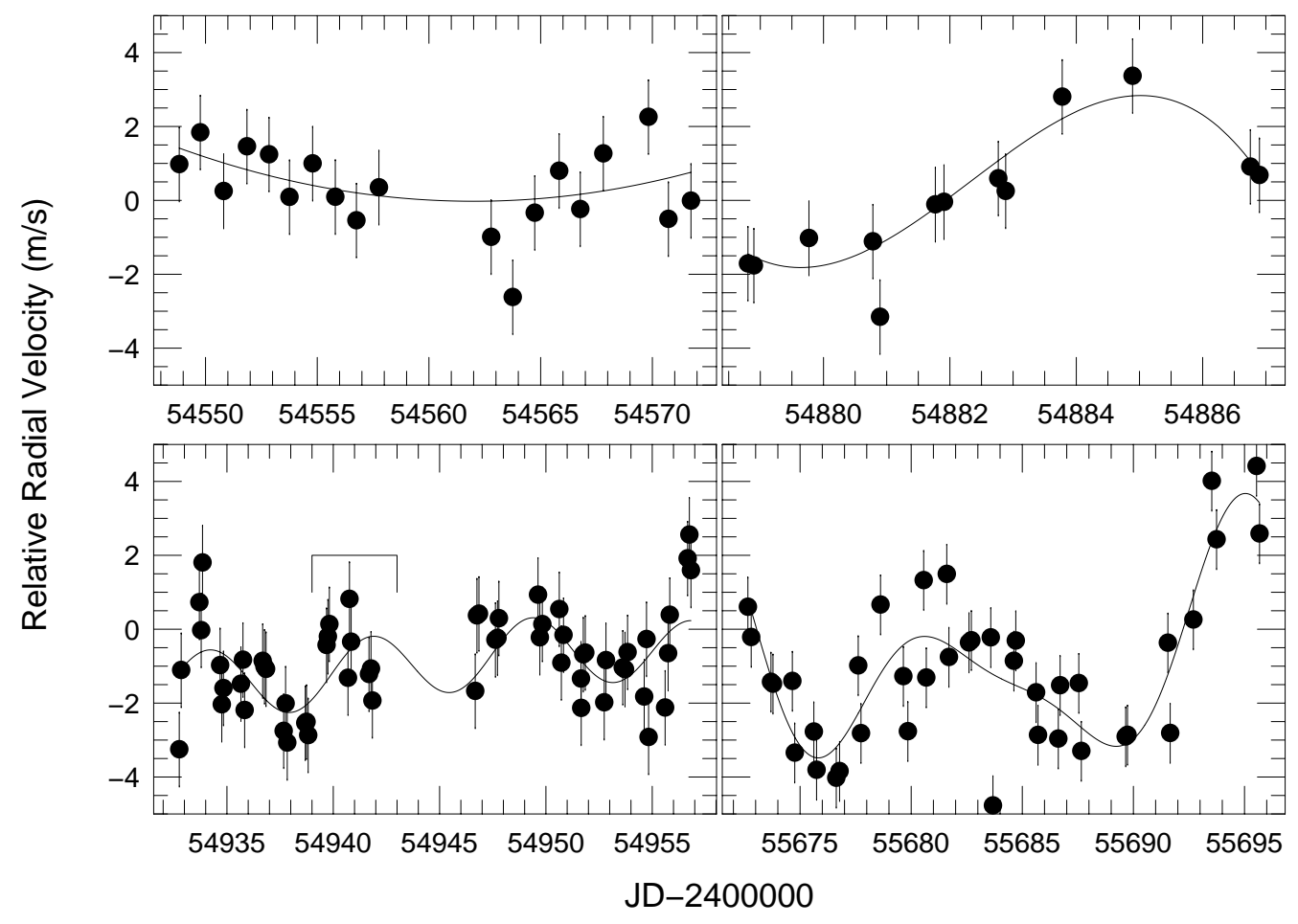

Fig. 12.- Time chunks and the underlying trend fits used in the second version of local trend filtering (LTF2). The bracket in the lower left panel shows points removed in the LTF1 analysis (see text). 


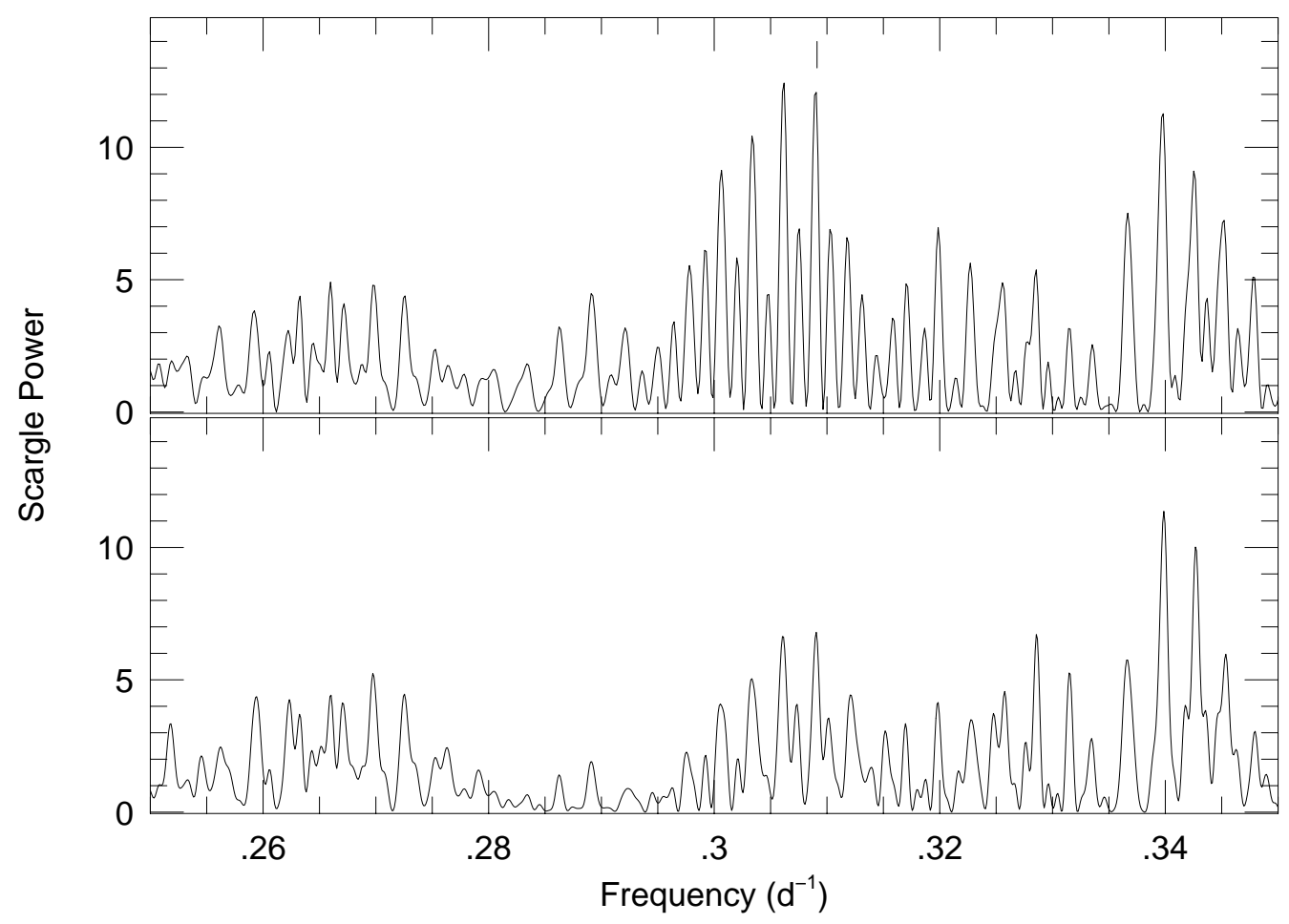

Fig. 13.- (Top) Periodogram of the RV residuals after applying the second local trend fitting (LFT2). The vertical line marks the orbital frequency of $\alpha$ Cen Bb. (Bottom) The periodogram the LFT2 residuals but with different filtering of the data in Chunk8-9. 


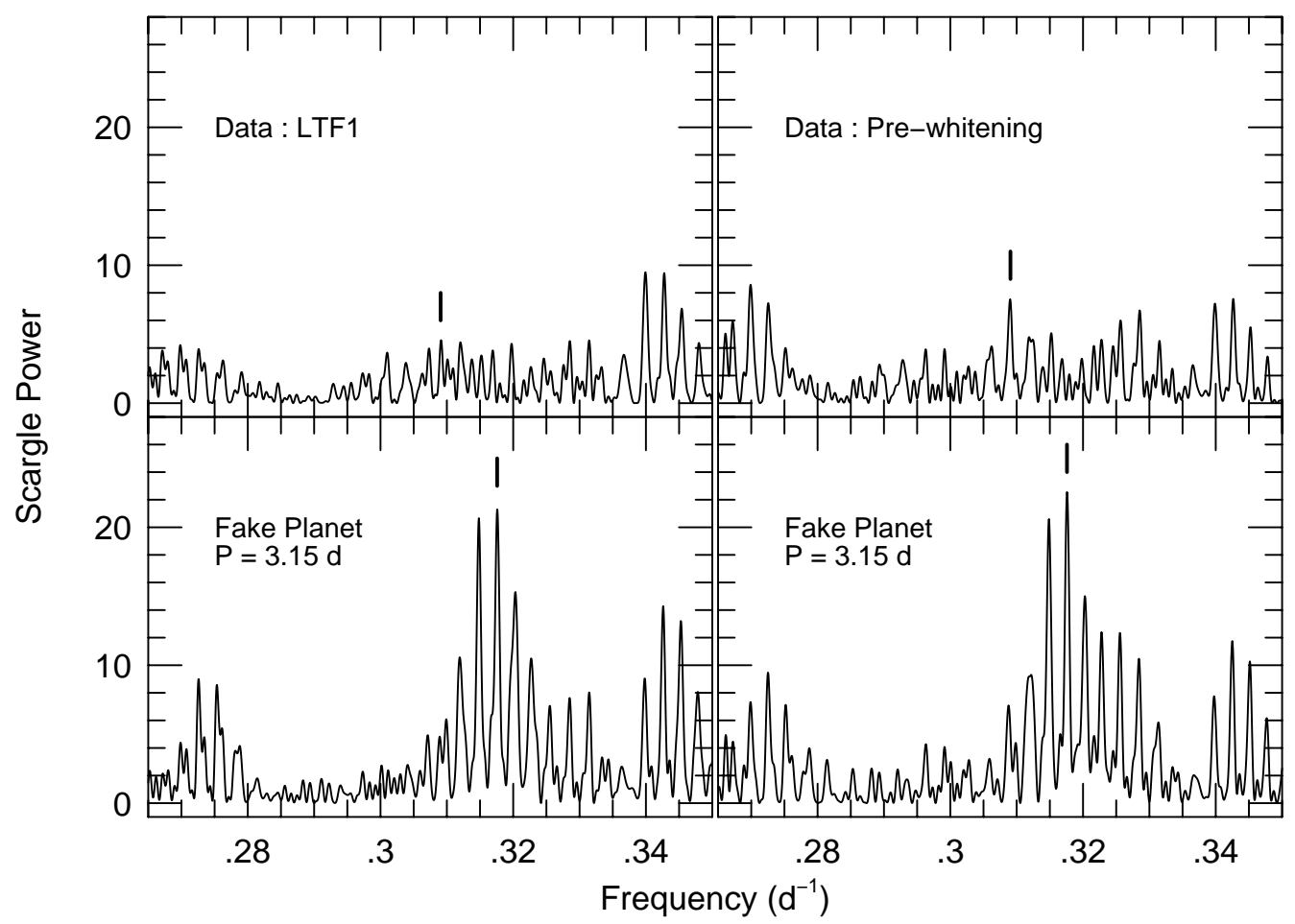

Fig. 14.- (Upper left panel) Scargle periodograms of the activity filtered RV data LTF1, but with Chunk8-9 removed. (Lower left panel) The Scargle periodogram of LTF1 filtered RV data minus Chunk8-9, but with an artificial planet signal $\left(P=3.15\right.$ days; $\nu=0.3175 \mathrm{~d}^{-1}$, $K=0.5 \mathrm{~m} \mathrm{~s}^{-1}$ ) inserted into the data prior to filtering. (Upper right) Scargle periodogram of the RV data without Chunk8-9 after filtering the activity signal with pre-whitening. (Lower right) Same as for the lower left panel (data with an artificial planet signal added) but for the pre-whitening procedure. The vertical line marks the location of the orbital frequency of $\alpha$ Cen $\mathrm{Bb}$ (top panels) or the simulated planet signal (lower panels). 


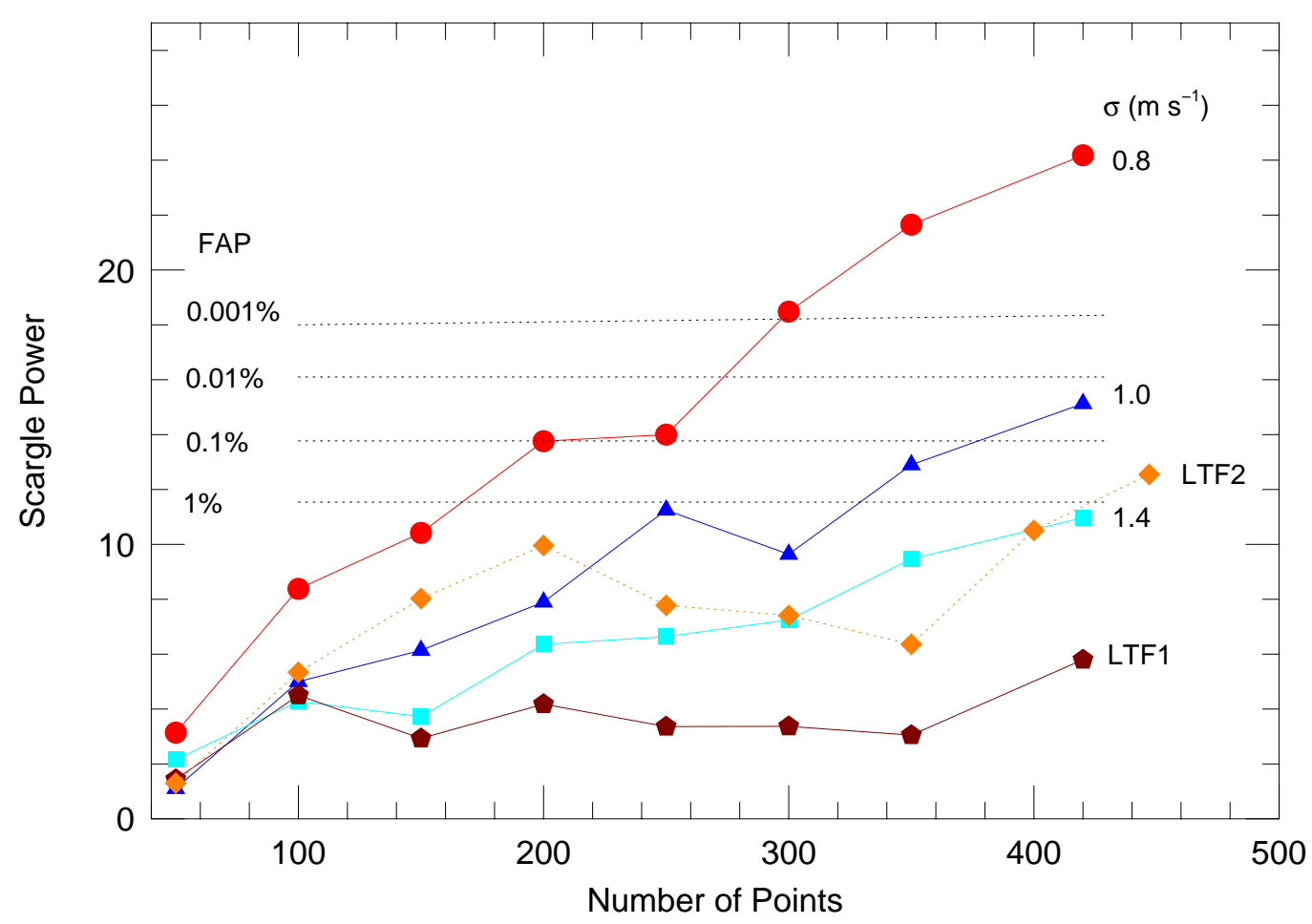

Fig. 15. - The Scargle power of the planet signal in the residual data from LTF1 (pentagons) and LTF2 (diamonds) as a function of number of data points. The same is shown for simulated data taking the activity function, a synthetic planet signal, and applying local trend fitting (time windows of LTF1). Noise has been added at three levels: $\sigma=0.8 \mathrm{~ms}^{-1}$ (dots), $1.0 \mathrm{~m} \mathrm{~s}^{-1}$ (triangles), and $1.4 \mathrm{~m} \mathrm{~s}^{-1}$ (squares). 


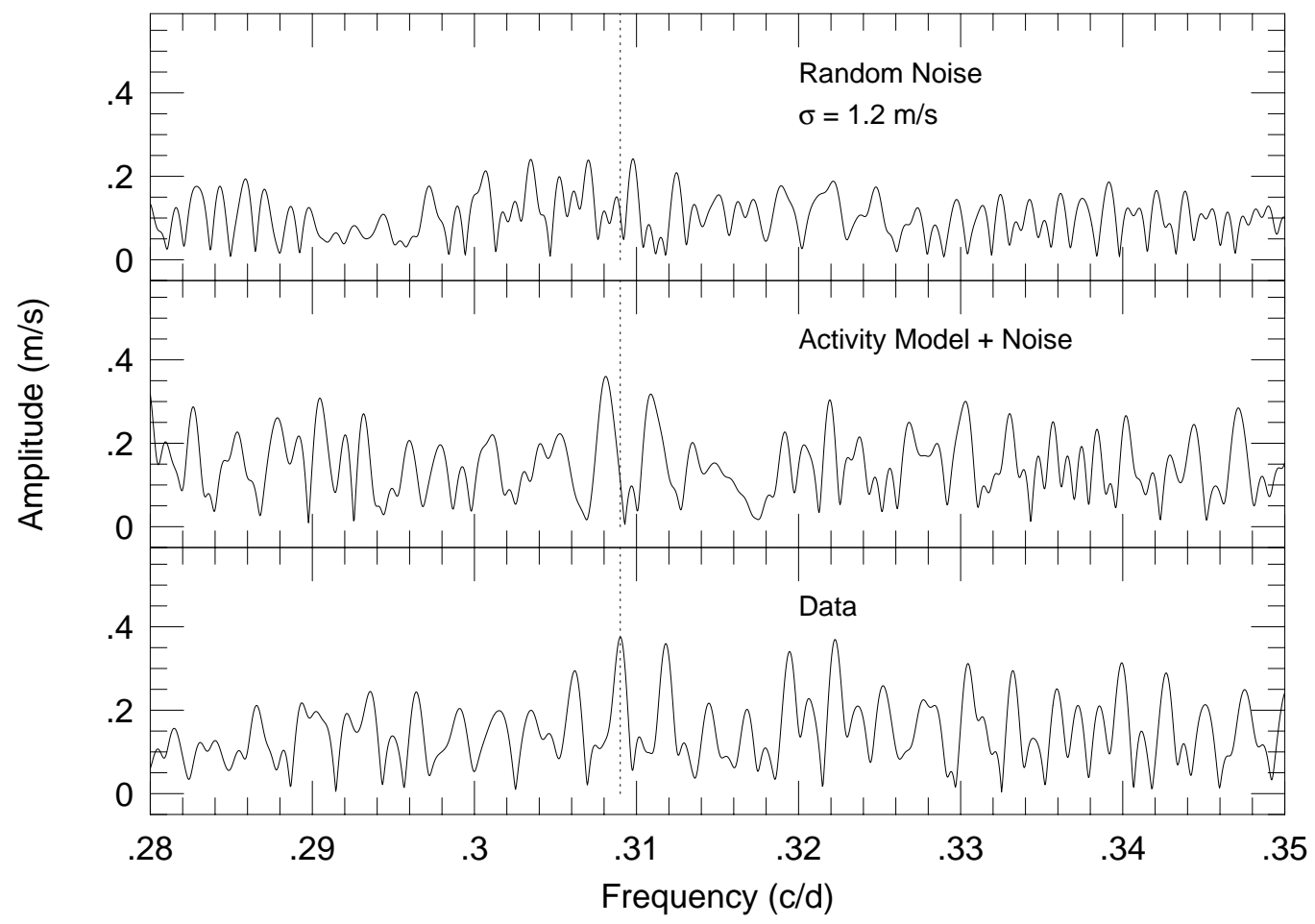

Fig. 16. - (Top) The Fourier amplitude spectrum of random noise with $\sigma=1.2 \mathrm{~m} \mathrm{~s}^{-1}$ and with the same time sampling as the data. (middle) The amplitude spectrum of the activity function with random noise $\left(\sigma=1.2 \mathrm{~m} \mathrm{~s}^{-1}\right)$ added. (Bottom) The Fourier amplitude spectrum of the actual unfiltered RV data. The dashed vertical line marks the planet orbital frequency. 


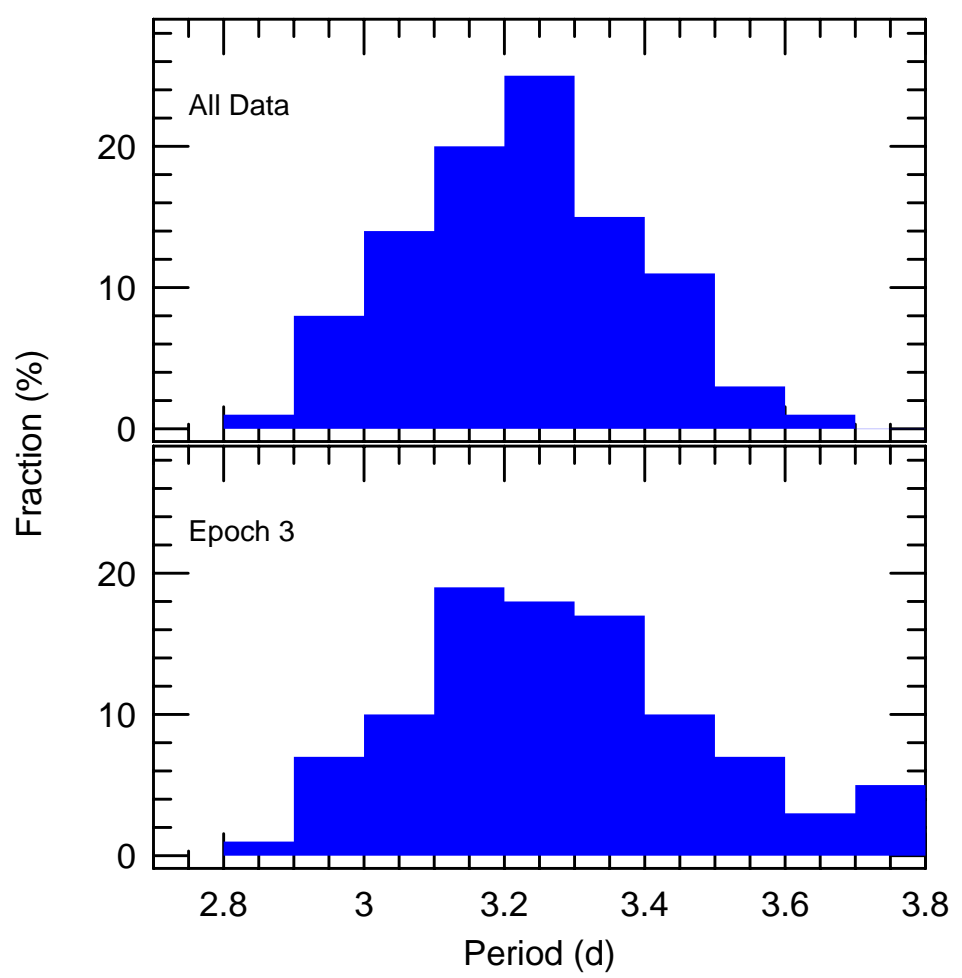

Fig. 17.- (Top) The distribution of the dominant noise peaks in the period range $P=2.8$ - $3.8 \mathrm{~d}$ for the full data set. Random noise data sampled in the same manner as the real data with $\sigma=1.2 \mathrm{~m} \mathrm{~s}^{-1}$ were used. The mean amplitude of the noise peaks is $K=0.24 \pm$ $0.04 \mathrm{~m} \mathrm{~s}^{-1}$. (Bottom) The same as the top panel but using only the time sampling window of Epoch 3. The mean amplitude of the noise peaks is $K=0.33 \pm 0.13 \mathrm{~m} \mathrm{~s}^{-1}$. 